\title{
Solving Asset Pricing Models with Stochastic Volatility
}

\author{
Oliver de Groot* \\ Federal Reserve Board
}

December 29, 2014

\begin{abstract}
This paper provides a closed-form solution for the price-dividend ratio in a standard asset pricing model with stochastic volatility. The growth rate of the endowment is a first-order Gaussian autoregression, while the stochastic volatility innovations can be drawn from any distribution for which the moment-generating function exists. The solution is useful in allowing comparisons among numerical methods used to approximate the nontrivial closed form. The closed-form solution reveals that, when using perturbation methods around the deterministic steady state, the approximate solution needs to be sixth-order accurate in order for the parameter capturing the conditional standard deviation of the stochastic volatility process to be present.
\end{abstract}

Keywords: Endowment model, Price-dividend ratio, Closed-form solution, Numerical methods.

JEL classifications: C61, C62, G12

*Affiliation: Board of Governors of the Federal Reserve System, Washington, D.C. Email: oliver.v.degroot@frb.gov. Disclaimer: The views expressed in this paper are those of the author and do not necessarily reflect those of the Board of Governors or staff of the Federal Reserve System. 


\section{Introduction}

Stochastic volatility has become an important feature of macroeconomic models that seek to explain both stylized business cycle and asset pricing facts. Since closed-form solutions elude richer macroeconomic models, various numerical methods have been proposed to provide an approximated solution. The contribution of this paper is to present a simple (asset pricing) stochastic volatility model in which an exact solution (for the pricedividend ratio) exists, which may serve as a benchmark from which to compare alternative numerical approximation methods.

Burnside (1998) provided an exact solution for the Lucas (1978) asset pricing model with Gaussian, autoregressive dividend growth shocks and time-separable constant relative risk-aversion (CRRA) preferences. ${ }^{1}$ Bidarkota and McCulloch (2003) and Tsionas (2003) extended Burnside's solution to shocks with stable distributions and shocks with well-defined moment-generating functions (MGFs), respectively, while Chen et al. (2008) and Collard et al. (2006) extended it to the case with non-time-separable preferences through habits in consumption. ${ }^{2}$ In each case, the solutions provide a useful benchmark against which to test numerical solution algorithms. This paper follows in that tradition. It extends the Burnside model by adding stochastic volatility to the dividend growth process.

Since Bansal and Yaron (2004) showed the importance of stochastic volatility to account for stylized asset pricing facts, the use of stochastic volatility has become a widespread addition to standard business cycle models. Yet, even beside the demand for business cycle models to match stylized asset pricing facts, there is a growing use of stochastic volatility in macro modelling. Stock and Watson (2002) and Sims and Zha (2006) are prominent examples arguing that time-varying volatility is important in accounting for the dynamics of U.S. aggregate data. Among Dynamic Stochastic General Equilibrium (DSGE) researchers, stochastic volatility is being put to many applications: Bloom et al. (2007) consider the role of time-varying uncertainty for investment dynamics, Justiniano and Primiceri (2008) investigate the sources of the Great Moderation, and Fernández-Villaverde et al. (2011) study the effects of stochastic volatility in fiscal shocks on economic activity, to name just a few.

Because of the increasing importance of stochastic volatility, which naturally adds additional nonlinearity into the solution of models, a growing literature has been testing how different numerical solution methods that solve equilibrium models with stochastic volatility perform. Caldara et al. (2012), for example, compare perturbation methods (of second and third order), Chebyshev polynomials, and value function iteration in a real

\footnotetext{
${ }^{1}$ An early contribution by Labadie (1989) also provided the solution in a slightly more general context.

${ }^{2}$ In related work, Calin et al. (2005) develop a method that finds the solutions for analytic utility functions that offer closed forms for a wide class of probability distributions for the state variable. Similarly, Le et al. (2010) extend the Gaussian dynamic term structure model to a larger class of MGFs.
} 
business cycle model with stochastic volatility.

In this paper, I show the exact solution for the price-dividend ratio of a simple asset pricing model as a nontrivial function of the model's two state variables, the current dividend growth rate and the current volatility of the dividend growth process. ${ }^{3}$ Innovations to the dividend growth rate are drawn from a Gaussian distribution. Innovations to the stochastic volatility process can be drawn from any distribution for which the MGF exists. For much of the paper, I follow Bansal and Yaron (2004) and assume Gaussian shocks for the stochastic volatility innovations. However, a gamma distribution is potentially appealing because it ensures that the realizations of the stochastic volatility process are strictly nonnegative and because it displays skewness and kurtosis.

The closed-form solution has the following properties: First, the price-dividend ratio increases when the volatility of dividend growth increases, as well as when the volatility of the stochastic volatility process increases. Second, the sensitivity of the price-dividend ratio to a change in the volatility state is increasing in the persistence of the stochastic volatility process. I derive an expression for the unconditional mean of the price-dividend process, as well as several other endogenous objects of interest, such as the risk-free rate and the conditional equity risk premium. Since the closed-form solution for the pricedividend ratio takes the form of an infinite sum, I provide parameter conditions under which the price-dividend ratio (and its unconditional mean) are finite. I also show where to truncate the infinite summation when calculating the solution numerically to ensure that the truncation error is no larger than a given value with a given probability.

Finally, I show how two alternative low-order polynomial approximation techniques perform in terms of numerical accuracy: (1) a first-order approximation following Campbell and Shiller (1988) that exploits the normality of the stochastic processes; and (2) the perturbation method around a deterministic steady state, popular among macro-DSGE researchers. I find two results of note: First, a fourth-order perturbation is required to generate a similar order of accuracy close to the steady state as the approximation that exploits the normality of the stochastic processes. Second, a sixth-order perturbation approximation is required for the parameter capturing the conditional standard deviation of the stochastic volatility process to show up in the approximated solution.

The rest of the paper is structured as follows. Section 2 presents the basic asset pricing model with stochastic volatility, and Section 3 presents the general closed-form solution. Section 4 applies the model and further discusses its uses. Section 5 concludes. The appendix provides derivations of the paper's key results, while an extensive online appendix provides additional detail, describes a variant of the basic model, and tests the model's asset pricing implications.

\footnotetext{
${ }^{3}$ The model features CRRA preferences and not recursive preferences as in Bansal and Yaron (2004), which means the model does not solve the risk-free rate and equity premium puzzles (see Mehra and Prescott (1985) and Weil (1989)). However, this feature does not diminish the model's usefulness as a testing ground for numerical solution methods interested in capturing the effects of stochastic volatility.
} 


\section{The asset pricing model}

There is a representative agent who maximizes the expected discounted stream of utility

$$
\mathbf{E}_{0} \sum_{t=0}^{\infty} \beta^{t} \frac{c_{t}^{1-\gamma}}{1-\gamma}
$$

subject to the budget constraint

$$
c_{t}+s_{t+1} p_{t} \leq\left(d_{t}+p_{t}\right) s_{t}
$$

where $\mathbf{E}_{t}$ is the mathematical expectations operator conditional on the time $t$ information set, $c_{t}$ is consumption, and $s_{t}$ denotes units of an asset whose price at date $t$ is $p_{t}$ with dividends $d_{t}$. The discount factor is $\beta \in(0,1)$, and the coefficient of relative risk aversion is $\gamma>0$ and $\gamma \neq 1$. The growth rate of dividends, denoted $x_{t} \equiv \log \left(d_{t} / d_{t-1}\right)$, is assumed to follow a Gaussian $A R(1)$ process,

$$
x_{t}=x+\rho\left(x_{t-1}-x\right)+\sqrt{\eta_{t}} \varepsilon_{t},
$$

where $x$ is the steady-state growth rate of dividends, $\rho \in(-1,1)$ is the persistence parameter, and $\varepsilon_{t}$ is a sequence of independently and identically distributed (iid) innovations from the standard normal distribution. The innovations to $x_{t}$ are scaled by $\sqrt{\eta_{t}}$. $\eta_{t}$ is therefore the conditional variance of dividend growth and is time varying. In particular, it follows an $A R(1)$ process,

$$
\eta_{t}=\eta+\rho_{\eta}\left(\eta_{t-1}-\eta\right)+\omega \varepsilon_{\eta, t}
$$

where $\eta$ is its steady state, $\rho_{\eta} \in(-1,1)$ is the persistence of the stochastic volatility process, $\omega$ is a scalar, and $\varepsilon_{\eta, t}$ is a sequence of iid innovations with a given distribution function $F_{\eta}$ and an MGF given by

$$
M(\tau) \equiv \mathbf{E} \exp \left(\tau \varepsilon_{\eta}\right)=\int_{-\infty}^{\infty} \exp \left(\tau \varepsilon_{\eta}\right) d F_{\eta}\left(\varepsilon_{\eta}\right), \quad \tau \in \mathbf{R}
$$

The first-order equilibrium condition of the agent's maximization problem, equations (1) and (2), is

$$
c_{t}^{-\gamma} p_{t}=\mathbf{E}_{t} \beta c_{t+1}^{-\gamma}\left(p_{t+1}+d_{t+1}\right) .
$$


Market clearing, $s_{t}=1$, implies that $c_{t}=d_{t},{ }^{4}$ and, in defining the price-dividend ratio as $y_{t} \equiv p_{t} / d_{t}$, the first-order equilibrium condition becomes

$$
y_{t}=\mathbf{E}_{t} \beta\left(\frac{d_{t+1}}{d_{t}}\right)^{1-\gamma}\left(y_{t+1}+1\right) .
$$

Iterating forward and making use of $x_{t}$, we are left with

$$
y_{t}=\sum_{i=1}^{\infty} \beta^{i} \mathbf{E}_{t} \exp \left((1-\gamma) \sum_{j=1}^{i} x_{t+j}\right)
$$

\section{The model solution}

Equation (7) shows that, in this asset pricing model, the price-dividend ratio at time $t$ is simply a function of expected future dividend growth. Finding an exact solution for $y_{t}$ means finding a closed-form expression for $\mathbf{E}_{t} \exp \left((1-\gamma) \sum_{j=1}^{i} x_{t+j}\right)$ for $i=1,2, \ldots$ in terms of the current state, $x_{t}$ and $\eta_{t}$. In the case without stochastic volatility, Burnside (1998) derived such a solution. The theorem below shows an exact solution with stochastic volatility.

Theorem 1 Suppose that the MGF of $\varepsilon_{\eta}$ exists. Then the solution to equation (7) is

$$
y_{t}=\sum_{i=1}^{\infty} \beta^{i} \exp \left(A_{i} x+B_{i}\left(x_{t}-x\right)+C_{i} \eta+D_{i}\left(\eta_{t}-\eta\right)+H_{i}\right),
$$

where

$$
\begin{gathered}
A_{i} \equiv(1-\gamma) i, \quad B_{i} \equiv\left(\frac{1-\gamma}{1-\rho}\right) \rho\left(1-\rho^{i}\right), \\
C_{i} \equiv \frac{1}{2}\left(\frac{1-\gamma}{1-\rho}\right)^{2}\left(i-2 \rho \frac{1-\rho^{i}}{1-\rho}+\rho^{2} \frac{1-\rho^{2 i}}{1-\rho^{2}}\right), \\
D_{i} \equiv \frac{\rho_{\eta}}{2}\left(\frac{1-\gamma}{1-\rho}\right)^{2}\left(\phi_{1}+\phi_{2} \rho_{\eta} \rho_{\eta}^{i-1}+\phi_{3} \rho^{i-1}+\phi_{4} \rho^{2(i-1)}\right), \\
H_{i} \equiv \sum_{j=1}^{i} \log M\left(\frac{\omega}{2}\left(\frac{1-\gamma}{1-\rho}\right)^{2}\left(\phi_{1}+\phi_{2} \rho_{\eta} \rho_{\eta}^{i-j}+\phi_{3} \rho^{i-j}+\phi_{4} \rho^{2(i-j)}\right)\right),
\end{gathered}
$$

and where

$$
\phi_{1} \equiv \frac{1}{1-\rho_{\eta}}, \quad \phi_{2} \equiv \frac{-\rho_{\eta}\left(\rho_{\eta}+\rho\right)(1-\rho)^{2}}{\left(\rho^{2}-\rho_{\eta}\right)\left(\rho-\rho_{\eta}\right)\left(1-\rho_{\eta}\right)}, \quad \phi_{3} \equiv \frac{-2 \rho^{2}}{\rho-\rho_{\eta}}, \quad \text { and } \quad \phi_{4} \equiv \frac{\rho^{4}}{\rho^{2}-\rho_{\eta}} .
$$

Proof. See Appendix A.1.

\footnotetext{
${ }^{4}$ The assumption that, in equilibrium, $c_{t}=d_{t}$ is not a necessary assumption to generate a closed-form solution. In the online Appendix B.4, I present a closed-form solution for the price-dividend ratio when the consumption and dividend growth processes follow the specificiation in Bansal and Yaron (2004). Specifically, in Bansal and Yaron (2004), consumption and dividends are driven by a common small predictable component but have (potentially) different steady-state growth rates and have independent iid innovations.
} 
Bansal and Yaron (2004) consider the case in which $\varepsilon_{\eta}$ is drawn from a Gaussian distribution, and I will focus on this case because of its near ubiquity in the finance literature. However, a drawback of the Gaussian distribution, though, is that it does not prevent the standard deviation of the dividend growth rate from turning negative. One solution to enforcing the nonnegativity constraint is to use a truncated normal distribution. A symmetrically truncated normal distribution, for example, has the following MGF:

$$
M^{T r N}(\tau)=\exp \left(\frac{\tau^{2}}{2}\right)\left(\frac{\Phi\left(-\varepsilon_{\eta}^{\min }-\tau\right)-\Phi\left(\varepsilon_{\eta}^{\min }-\tau\right)}{1-2 \Phi\left(\varepsilon_{\eta}^{\min }\right)}\right)
$$

where $\varepsilon_{\eta}^{\min }$ denotes the least restrictive truncation that ensures nonnegativity of $\eta_{t}\left(\varepsilon_{\eta}^{\min }\right.$ is explicitly defined in the online Appendix B.1.1). Alternatively, one could model $\varepsilon_{\eta}$ as drawn from a distribution with a nonnegative support but with an existing MGF, such as the gamma distribution. ${ }^{5}$ The gamma distribution has the following MGF:

$$
M^{\Gamma}(\tau)=\left(1-g_{1} \tau\right)^{-g_{2}} \quad \text { for } \quad \tau<\frac{1}{g_{1}}
$$

where $g_{1}$ and $g_{2}$ are the scale and shape parameter, respectively. The value of a closedform solution with the gamma distribution for the stochastic volatility innovations as opposed to the Gaussian distribution - is that there is plenty of empirical evidence (see for example, Geweke (1994) and Gallant et al. (1997)) in favor of fat tails in the stochastic volatility process for many financial series. The gamma distribution features both positive skew and excess kurtosis. Despite this fact, it is beyond the scope of the current paper to explore this avenue of research in more detail.

The result in Theorem 1 nests the solution for the model when $\varepsilon_{\eta}$ is drawn from a standard normal distribution, with an MGF of $M^{N}(\tau)=\exp \left(\frac{\tau^{2}}{2}\right)$, which I show in the following corollary: ${ }^{6}$

Corollary 2 When $\varepsilon_{\eta} \sim$ N.i.d. $(0,1)$, then $H_{i}$ in the solution in equation (8) becomes $F_{i} \omega^{2}$, where

$$
F_{i} \equiv \frac{1}{8}\left(\frac{1-\gamma}{1-\rho}\right)^{4}\left(\begin{array}{c}
i \phi_{1}^{2}+\phi_{2}^{2} \frac{1-\rho_{\eta}^{2 i}}{1-\rho_{\eta}^{2}}+\phi_{3}^{2} \frac{1-\rho^{2 i}}{1-\rho^{2}}+\phi_{4}^{2} \frac{1-\rho^{4 i}}{1-\rho^{4}} \\
+2 \phi_{1} \phi_{2} \frac{1-\rho_{\eta}^{i}}{1-\rho_{\eta}}+2 \phi_{1} \phi_{3} \frac{1-\rho^{i}}{1-\rho}+2 \phi_{1} \phi_{4} \frac{1-\rho^{2 i}}{1-\rho^{2}} \\
+2 \phi_{2} \phi_{3} \frac{1-\left(\rho_{\eta} \rho\right)^{i}}{1-\rho_{\eta} \rho}+2 \phi_{2} \phi_{4} \frac{1-\left(\rho_{\eta} \rho^{2}\right)^{i}}{1-\rho_{\eta} \rho^{2}}+2 \phi_{3} \phi_{4} \frac{1-\rho^{3 i}}{1-\rho^{3}}
\end{array}\right)
$$

\footnotetext{
${ }^{5}$ The inverse-gamma, another distribution popular in the finance stochastic volatility literature, does not have a well defined MGF.

${ }^{6}$ This formulation of the stochastic volatility process could technically cause the standard deviation of dividend growth to become negative. However, under reasonable calibrations of the process, this result happens rarely. Bansal and Yaron (2004) use the same process and choose the following parameter values based on a monthly frequency: $\eta=6.08 \times 10^{-5}, \rho_{\eta}=0.987$, and $\omega=0.23 \times 10^{-5}$. Simulating this process $10^{5}$ times for 840 months results in the process turning negative in $0.14 \%$ of the simulations.
} 
Proof. See Appendix A.2.

For the remainder of the paper, unless otherwise stated, I will be using the model with Gaussian stochastic volatility. In Burnside (1998), the solution without stochastic volatility is

$$
y_{t}=\sum_{i=1}^{\infty} \beta^{i} \exp \left(A_{i} x+B_{i}\left(x_{t}-x\right)+C_{i} \eta\right)
$$

therefore, it is the term $D_{i}\left(\eta_{t}-\eta\right)+F_{i} \omega^{2}$ inside the exponential function in equation (8) that is novel. It is straightforward to show (see equations (A.5) and (A.11) in Appendix A.1) that both $D_{i}>0$ and $F_{i}>0 .{ }^{7}$ It follows that $\frac{\partial y_{t}}{\partial\left(\eta_{t}-\eta\right)}>0$ and $\frac{\partial y_{t}}{\partial \omega^{2}}>0:$ A rise in the volatility of dividend growth unambiguously increases the price-dividend ratio, as does a rise in the volatility of the stochastic volatility process itself. This outcome is mechanically a consequence of the standard result that a rise in the volatility of a log-normal process increases its mean. It also follows that $\frac{\partial\left|\partial y_{t} / \partial\left(x_{t}-x\right)\right|}{\partial\left(\eta_{t}-\eta\right)}>0$ and $\frac{\partial\left|\partial y_{t} / \partial\left(x_{t}-x\right)\right|}{\partial \omega^{2}}>0$ : The price-dividend ratio responds more to movements in the dividend growth rate in a highvolatility state than in a low-volatility state, as well as in an environment with greater stochastic volatility. The insight from this result is that the heteroskedasticity (inherent in the exogenous dividend growth process) will be more pronounced in the endogenous price-dividend ratio. Equations (A.5) and (A.11) also show clearly that $\frac{\partial D_{i}}{\partial \rho_{\eta}}, \frac{\partial F_{i}}{\partial \rho_{\eta}}>0$ : A rise in the persistence of the stochastic volatility process increases the sensitivity of the price-dividend ratio to both changes in dividend growth and volatility.

\section{Application and further discussion}

This section presents several applications of the closed-form solution derived in Section 3. First, I derive closed-form expressions for several other variables of interest, such as the unconditional mean price-dividend ratio, the risk-free rate, the conditional-expected return on equity and the conditional equity risk premium. Second and third, since the price-dividend ratio is the sum of an infinite sequence, I show the conditions under which the price-dividend ratio exits (i.e. is finite) and I show how to choose an appropriate truncation point when calculating the price-dividend ratio. Fourth, I use the model to compare the numerical accuracy of several low-order polynomial approximations in capturing the effects of stochastic volatility.

\footnotetext{
${ }^{7}$ The exception is logarithmic preferences $(\gamma=1)$ in which case $A_{i}=B_{i}=C_{i}=D_{i}=F_{i}=0$ and the price-dividend ratio becomes constant. With logarithmic preferences, $B_{i}=0$ because the wealth and substitution effects of a change in the dividend growth rate exactly offset each other. Since the price-dividend ratio remains constant in response to dividend growth movements, it follows that the price-dividend ratio is also invariant to changes in the volatility of those movements.
} 


\subsection{Additional variables of interest}

Once we have a closed-form solution for the price-dividend ratio in hand, it is possible to construct closed-form expressions for several other variables of interest, including the unconditional mean price-dividend ratio, risk-free rate, conditional-expected return on equity, and the conditional equity risk premium. Derivations are contained in the online Appendix B.2.

Unconditional mean price-dividend ratio The unconditional mean price-dividend ratio is

$\mathbf{E} y_{t}=\sum_{i=1}^{\infty} \beta^{i} \exp \left(A_{i} x+\left(C_{i}+\frac{B_{i}^{2}}{2\left(1-\rho^{2}\right)}\right) \eta+\left(\frac{1}{2}\left(\frac{\gamma_{i, 1}^{2}}{1-\rho_{\eta}^{2}}-\frac{2 \gamma_{i, 1} \gamma_{i, 2}}{1-\rho_{\eta} \rho^{2}}+\frac{\gamma_{i, 2}^{2}}{1-\rho^{4}}\right)+F_{i}\right) \omega^{2}\right)$,

where

$$
\gamma_{i, 1} \equiv\left(\frac{B_{i}^{2}}{2} \frac{\rho_{\eta}}{\rho_{\eta}-\rho^{2}}+D_{i}\right), \quad \gamma_{i, 2} \equiv \frac{B_{i}^{2}}{2} \frac{\rho^{2}}{\rho_{\eta}-\rho^{2}}
$$

The unconditional mean price-dividend ratio is increasing in both the volatility $\omega$ and the persistence $\rho_{\eta}$ of the stochastic volatility process (as is made clear by the quadratic expression in (B.3) in Appendix B.2.1). The unconditional mean price dividend-ratio is also higher than the price-dividend ratio evaluated at the steady state $\left(x_{t}, \eta_{t}\right)=(x, \eta)$.

Risk-free rate The risk-free rate is defined as

$$
R_{t}^{r f}=\left(\mathbf{E}_{t}\left(\beta\left(\frac{c_{t+1}}{c_{t}}\right)^{-\gamma}\right)\right)^{-1}
$$

and has the following solution:

$$
R_{t}^{r f}=\beta^{-1} \exp \left(\gamma x+\gamma \rho\left(x_{t}-x\right)-\frac{\gamma^{2}}{2} \eta-\frac{\gamma^{2} \rho_{\eta}}{2}\left(\eta_{t}-\eta\right)-\frac{\gamma^{4}}{8} \omega^{2}\right)
$$

Higher dividend growth increases the risk-free rate, while higher stochastic volatility lowers the risk-free rate. Thus, the risk-free rate puzzle (see Weil (1989)) may be partly resolved by the addition of stochastic volatility. ${ }^{8}$ As is typical in this class of models, when the risk-aversion parameter, $\gamma$ is large, the risk-free rate can become counterfactually high.

\footnotetext{
${ }^{8}$ In online Appendix B.5, I quantitatively assess the ability of stochastic volatility to lower the risk-free rate. In this model with CRRA preferences, a counterfactually large standard deviation of the stochastic volatility innovations is required to generate an economically meaningful reduction in the risk-free rate.
} 
Conditional-expected return on equity and the equity risk premium The conditional-expected return on equity is defined as

$$
\mathbf{E}_{t} R_{t+1}=\mathbf{E}_{t}\left(\frac{d_{t+1}+p_{t+1}}{p_{t}}\right)
$$

and can be rewritten as

$$
\mathbf{E}_{t} R_{t+1}=\frac{\mathbf{E}_{t} \exp \left(x_{t+1}\right)+\mathbf{E}_{t} y_{t+1} \exp \left(x_{t+1}\right)}{y_{t}} .
$$

The solution replaces $\mathbf{E}_{t} \exp \left(x_{t+1}\right)$ with

$$
\exp \left(x+\rho\left(x_{t}-x\right)+\frac{1}{2} \eta+\frac{\rho_{\eta}}{2}\left(\eta_{t}-\eta\right)+\frac{1}{8} \omega^{2}\right)
$$

and $\mathbf{E}_{t} y_{t+1} \exp \left(x_{t+1}\right)$ with

$$
\sum_{i=1}^{\infty} \beta^{i} \exp \left(\begin{array}{c}
\left(A_{i}+1\right) x+\left(B_{i}+1\right) \rho\left(x_{t}-x\right) \\
+\left(C_{i}+\frac{1}{2}\left(B_{i}+1\right)^{2}\right) \eta+\frac{1}{2}\left(B_{i}+1\right)^{2} \rho_{\eta}\left(\eta_{t}-\eta\right) \\
+\left(F_{i}+\frac{1}{2}\left(\frac{1}{2}\left(B_{i}+1\right)^{2}+D_{i}\right)^{2}\right) \omega^{2}
\end{array}\right)
$$

Since the conditional-expected return on equity is the ratio of two objects involving a sum of exponential terms in $x_{t}$ and $\eta_{t}$, it is, unfortunately, not possible to derive a closed-form expression for the unconditional mean return on equity or for the unconditional equity risk premium. The conditional-equity risk premium is $\mathbf{E}_{t} R_{t+1}-R_{t}^{r f}$.

\subsection{Existence}

Since the price-dividend ratio is the sum of an infinite sequence, it is not clear from equation (8) whether the price-dividend ratio is finite. The following theorem states the parameter conditions under which the price-dividend ratio $i s$ finite:

Theorem 3 The series in equation (8) with $H_{i}=F_{i} \omega^{2}$ converges if and only if

$$
\beta \exp \left((1-\gamma) x+\frac{1}{2}\left(\frac{1-\gamma}{1-\rho}\right)^{2} \eta+\frac{(1-\gamma)^{4}}{8(1-\rho)^{4}\left(1-\rho_{\eta}\right)^{2}} \omega^{2}\right)<1 \text {. }
$$

Proof. See Appendix A.3.

In Burnside (1998), the convergence criterion is

$$
\beta \exp \left((1-\gamma) x+\frac{1}{2}\left(\frac{1-\gamma}{1-\rho}\right)^{2} \eta\right)<1
$$


and thus less demanding than the condition in Theorem 3, conditional on the same parameters for $\beta, \gamma, x, \rho$, and $\eta{ }^{9}$

To get a better understanding of the restriction the condition in Theorem 3 places on the parameters of the stochastic volatility process, I followed Schmitt-Grohé and Uribe (2004) and Bansal and Yaron (2004) in parameterizing the asset pricing model as follows: Based on an annual frequency, $\beta=0.95, x=0.0179$, and $\eta=0.0012$. In addition, I consider three different parameterizations of the pair $(\rho, \gamma)$ using $\rho=\{-0.137,0.868\}$ and $\gamma=\{2.5,21\}$. I ignore the high-persistence, high-risk-aversion combination since the price-dividend ratio is never finite in this case. Figure 1 shows the $\left(\rho_{\eta}, \omega\right)$ pairs (the two parameters describing the stochastic volatility process) for which the condition for a finite price-dividend ratio (in Theorem 3) holds. The plots show that, when both the persistence of the endowment growth process and risk aversion are low (left panel), the conditions on the stochastic volatility process to ensure that the price-dividend ratio is finite are relatively weak. Bansal and Yaron (2004) choose parameter values for $\rho_{\eta}$ and $\omega$ (indicated in the figure), significantly inside the convergent parameter space. ${ }^{10}$ However, as either the level of risk aversion (middle panel) or the persistence of the dividend growth process (right panel) increases, the parameter space for the stochastic volatility process consistent with a finite price-dividend ratio shrinks considerably.

\subsection{Accuracy of calculating the price-dividend ratio}

Despite a closed-form solution, the solution in equation (8) is that of an infinite sum, which means that when the model is calibrated and the price-dividend ratio is calculated, some level of truncation, and therefore inaccuracy, is inevitable. Here I show how to choose an appropriate truncation point. Denote $y_{t}^{N}$ as the truncated solution

$$
y_{t}^{N}=\sum_{i=1}^{N} \beta^{i} \exp \left(Z_{i}+B_{i}\left(x_{t}-x\right)+D_{i}\left(\eta_{t}-\eta\right)\right)
$$

where, for parsimony, $Z_{i} \equiv A_{i} x+C_{i} \eta+F_{i} \omega^{2}$. Then select $N$ such that $\mathbf{P}\left(\Delta y_{t}^{N} \geq \xi\right) \leq \psi$, where $\Delta y_{t}^{N} \equiv y_{t}^{N}-y_{t}^{N-1}$ and $\xi, \psi>0$. In words, select a truncation point $N$ such that the probability of an error greater than some value $\xi$ is smaller than some probability $\psi$. Since

$$
\Delta y_{t}^{N}=\beta^{N} \exp \left(Z_{N}+B_{N}\left(x_{t}-x\right)+D_{N}\left(\eta_{t}-\eta\right)\right)
$$

Markov's inequality implies that

$\mathbf{P}\left(\Delta y_{t}^{N} \geq \xi\right)<\frac{\mathbf{E}\left(\Delta y_{t}^{N}\right)}{\xi}=\frac{\beta^{N}}{\xi} \exp \left(Z_{N}+\frac{1}{2} \frac{B_{N}^{2} \eta}{1-\rho^{2}}+\frac{\omega^{2}}{2}\left(\frac{\gamma_{N, 1}^{2}}{1-\rho_{\eta}^{2}}-\frac{2 \gamma_{N, 1} \gamma_{N, 2}}{1-\rho_{\eta} \rho^{2}}+\frac{\gamma_{N, 2}^{2}}{1-\rho^{4}}\right)\right)$.

\footnotetext{
${ }^{9}$ It is straightforward to show that the unconditional mean price-dividend ratio is also finite if and only if the condition in Theorem 3 holds (see the online Appendix B.2.1).

${ }^{10}$ Bansal and Yaron (2004) use a monthly calibration with $\rho_{\eta}=0.987$ and $\omega=0.23 \times 10^{-5}$. Figure 1 reports the annualized-equivalent values of $\rho_{\eta}=0.855$ and $\omega=0.74 \times 10^{-5}$.
} 
Figure 1: Regions of convergence in the parameter space
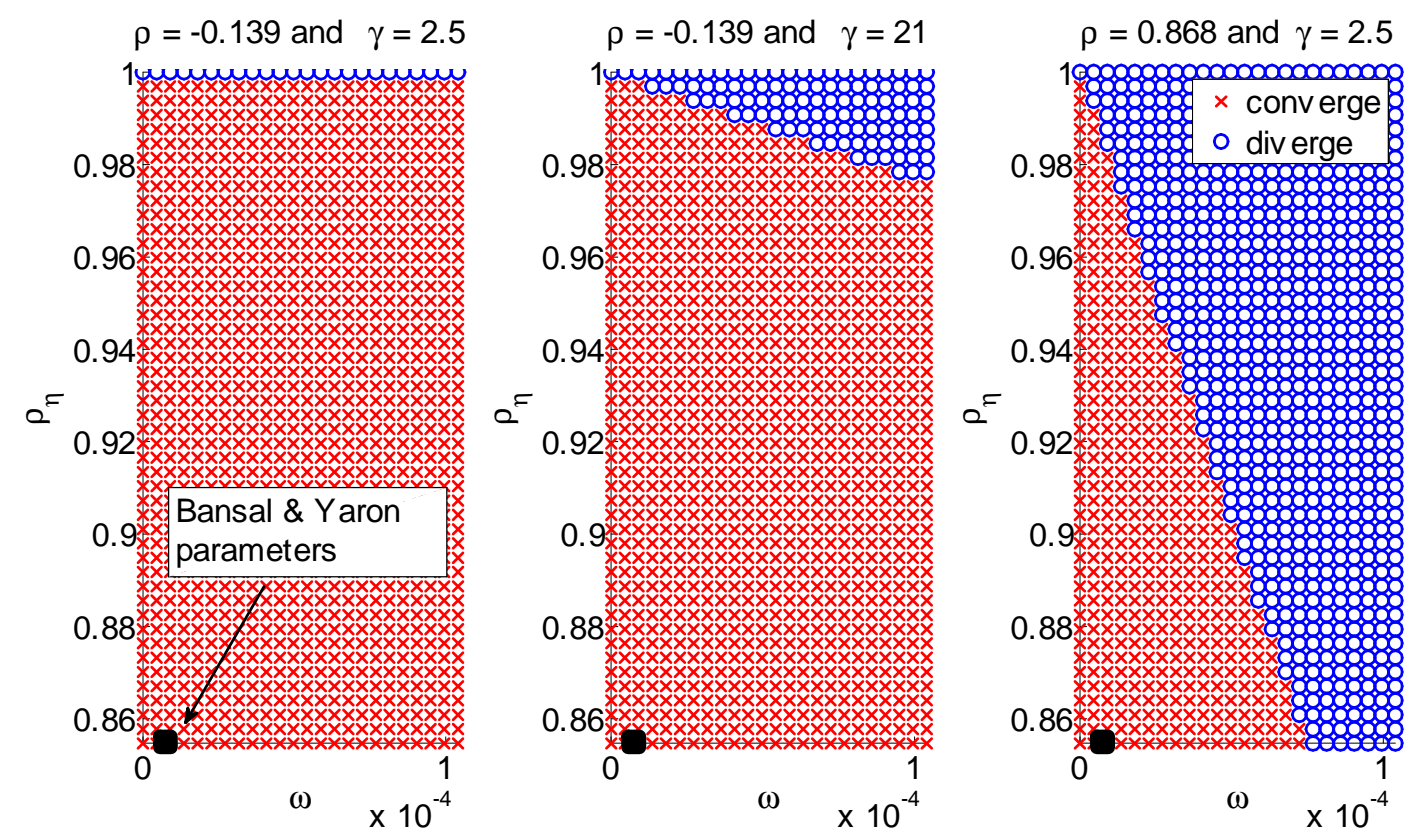

Note: Red crosses mark the parameter space for which the condition in Theorem 3 holds, blue circles the parameter space for which the condition is violated and the price-dividend ratio is no longer finite. The black square denotes parameters values $\rho_{\eta}=0.855$, and $\omega=0.74 \times 10^{-5}$ used by Bansal and Yaron (2004). Remaining parameters are $\beta=0.95, x=0.0179$ and $\eta=0.0012$. 
It is then possible to select an $N$ such that

$$
\frac{\beta^{N}}{\xi} \exp \left(Z_{N}+\frac{1}{2} \frac{B_{N}^{2} \eta}{1-\rho^{2}}+\frac{\omega^{2}}{2}\left(\frac{\gamma_{N, 1}^{2}}{1-\rho_{\eta}^{2}}-\frac{2 \gamma_{N, 1} \gamma_{N, 2}}{1-\rho_{\eta} \rho^{2}}+\frac{\gamma_{N, 2}^{2}}{1-\rho^{4}}\right)\right)<\psi,
$$

where $\xi$ can be set to machine precision and $\psi$ can be a desirably low probability.

\subsection{Low-order polynomial approximations}

While the model presented in this paper is too stylized to provide a strong quantitative description of asset prices, the closed-form solution of the model provides a useful benchmark to compare the properties of numerical solution techniques that one may wish to employ for richer, quantitative models with stochastic volatility. As an example, this section compares the results from a perturbation solution around the deterministic steady state (up to sixth order) with a linear approximation that exploits the Gaussian nature of the shocks.

\subsubsection{The perturbation solution}

Perturbation methods, popular in macro-DSGE models, create a polynomial approximation around the deterministic steady state. Since Schmitt-Grohé and Uribe (2004) show that perturbation methods generate accurate approximations, I can work directly with a Taylor expansion around the known decision rule. ${ }^{11}$ The perturbation method incorporates a scale parameter, which I denote $\sigma$, that scales the stochastic processes in the model. When $\sigma=0$, we have the deterministic counterpart of the model, and, when $\sigma=1$, we have the full stochastic model. The solution of the model, with the inclusion of the perturbation parameter, can therefore be rewritten as

$$
y_{t}=\sum_{i=1}^{\infty} \beta^{i} \exp \left(A_{i} x+B_{i}\left(x_{t}-x\right)+C_{i} \sigma^{2} \eta+D_{i} \sigma^{2}\left(\eta_{t}-\eta\right)+F_{i} \sigma^{6} \omega^{2}\right),
$$

and, in general, the decision rule can written as

$$
y_{t}=g\left(x_{t}, \eta_{t}, \sigma\right)
$$

The perturbation method constructs a polynomial expansion of $g\left(x_{t}, \eta_{t}, \sigma\right)$ around $\left(x_{t}, \eta_{t}, \sigma\right)=$ $(x, \eta, 0)$. Since the following coefficients, when evaluated at the deterministic steady-state, are all zero

$$
\begin{gathered}
g_{\eta}=g_{\sigma}=g_{\eta^{2}}=g_{x \eta}=g_{x \sigma}=g_{\eta \sigma}=0 \\
g_{\eta^{3}}=g_{\sigma^{3}}=g_{x^{2} \eta}=g_{x^{2} \sigma}=g_{\eta^{2} x}=g_{\eta^{2} \sigma}=g_{x \eta \sigma}=0
\end{gathered}
$$

\footnotetext{
${ }^{11}$ In this instance, accuracy means that the perturbed approximate solution around the deterministic steady state delivers the same decision rule as if one had done a Taylor expansion of the exact decision rule.
} 
the third-order solution is

$$
\begin{gathered}
y_{t}=g+g_{x}\left(x_{t}-x\right)+\frac{1}{2}\left(g_{x^{2}}\left(x_{t}-x\right)^{2}+g_{\sigma^{2}}\right) \\
+\frac{1}{6}\left(g_{x^{3}}\left(x_{t}-x\right)^{3}+3 g_{\sigma^{2} x}\left(x_{t}-x\right)+3 g_{\sigma^{2} \eta}\left(\eta_{t}-\eta\right)\right),
\end{gathered}
$$

where, for example, $g_{\sigma^{2} x} \equiv \frac{\partial^{3} g(x, \eta, 0)}{\partial \sigma^{2} \partial x}$. The non-zero coefficients are

$$
\begin{gathered}
g_{x}=\sum_{i=1}^{\infty} \beta^{i} \exp \left(A_{i} x\right) B_{i}, \\
g_{x^{2}}=\sum_{i=1}^{\infty} \beta^{i} \exp \left(A_{i} x\right) B_{i}^{2}, \quad g_{\sigma^{2}}=\sum_{i=1}^{\infty} \beta^{i} \exp \left(A_{i} x\right) 2 \eta C_{i}, \\
g_{x^{3}}=\sum_{i=1}^{\infty} \beta^{i} \exp \left(A_{i} x\right) B_{i}^{3}, \quad g_{\sigma^{2} x}=\sum_{i=1}^{\infty} \beta^{i} \exp \left(A_{i} x\right) 2 \eta B_{i} C_{i}, \quad g_{\sigma^{2} \eta}=\sum_{i=1}^{\infty} \beta^{i} \exp \left(A_{i} x\right) 2 D_{i} .
\end{gathered}
$$

It is a well known result in the macro literature, that a third-order approximation around the deterministic steady state is required for the first-order effects of stochastic volatility to appear. That is, the first occurrence of $\left(\eta_{t}-\eta\right)$ in the approximate solution, equation (11), is for the third order term $g_{\sigma^{2} \eta}$. Less well known is the following theorem:

Theorem 4 The price-dividend ratio in a model with a dividend growth process described by (3) and (4) is only affected by the standard deviation of the stochastic volatility process, $\omega$ if the perturbed approximation around the deterministic steady state is taken up to sixth order.

The coefficients of the approximations for fourth to sixth orders are unwieldy and are therefore relegated to the online Appendix B.3.1. However, the parameter $\omega$ is absent in all the terms except for

$$
g_{\sigma^{6}}=\sum_{i=1}^{\infty} \beta^{i} \exp \left(A_{i} x\right)\left(\eta^{3} C_{i}^{3}+720 F_{i} \omega^{2}\right)
$$

This result is also clear from equation (10) since the perturbation parameter raised to the power six, $\sigma^{6}$, premultiplies $\omega^{2}$. While the role of $\omega$ is not very powerful in this simple model with CRRA utility, in Bansal and Yaron (2004), the addition of stochastic volatility is crucial in raising the mean equity premium from $4.20 \%$ to $6.84 \%$ in their preferred specification. Theorem 4 may explain some of the relative success of endowment economy models like Bansal and Yaron (2004) versus production economy models in capturing the observed equity risk premium. Bansal and Yaron (2004) show that adding stochastic volatility to a model with recursive preferences can significantly increase the equity risk premium. In contrast, when Andreasen (2012) adds stochastic volatility to the New Keynesian model with recursive preferences presented by Rudebusch and Swanson (2012), he still requires a coefficient of relative risk aversion in excess of 150 in order to match observed term premiums. In both papers, the model is solved using perturbation 
methods up to only third order. ${ }^{12}$

\subsubsection{The first-order solution exploiting normality}

An alternative to perturbation methods is to follow Campbell and Shiller (1988) and to exploit the conditional-log-normality of the dividend growth process. The detailed derivation is in the online Appendix B.3.2. The log-price-dividend ratio in this case is specified as

$$
\log y_{t}=\log y+\kappa_{1}\left(x_{t}-x\right)+\kappa_{2}\left(\eta_{t}-\eta\right)
$$

where

$$
\kappa_{1} \equiv \frac{(1-\gamma) \rho}{1-\frac{y}{1+y} \rho}, \quad \kappa_{2} \equiv \frac{\frac{1}{2}\left((1-\gamma)+\frac{y}{1+y} \kappa_{1}\right)^{2} \rho_{\eta}}{1-\frac{y}{1+y} \rho_{\eta}}
$$

and $y$ solves

$$
\frac{y}{1+y}=\beta \exp \left((1-\gamma) x+\frac{(1-\gamma)^{2}(1+y)^{2} \eta}{2(1+(1-\rho) y)^{2}}+\frac{(1-\gamma)^{4}(1+y)^{6} \omega^{2}}{8(1+(1-\rho) y)^{4}\left(1+\left(1-\rho_{\eta}\right) y\right)^{2}}\right) .
$$

With the approximate solutions using the two alternative methods in hand, it is possible to compare accuracy relative to the closed-form solution, which can be calculated to machine precision. Figure 2 presents accuracy results using the benchmark calibration employed in the previous subsection (with $(\rho, \gamma)=(-0.137,2.5)$ ). Since the state space is two dimensional, I present several different cuts of the accuracy statistic. The top row shows the accuracy of the price-dividend ratio as $x_{t}$ changes, holding $\eta_{t}$ fixed at $0, \eta$, and $4 \eta$, respectively, as one moves from the left to right panels. When comparing the left and right panels with the middle panel, the second-order approximation does poorly when the volatility of the dividend growth process is away from its steady state. Looking across all six panels, it is clear that the Campbell and Shiller (1988) approximation (denoted c-s approx.) does very well. A fourth-order solution performs well close to the steady state, but deteriorates relative to the Campbell and Shiller (1988) approximation as $\eta_{t}$ moves further from $\eta$. Following Theorem 4, it is also not surprising that a sixth-order approximation generates another large improvement in terms of accuracy (the fifth-order approximation was excluded, as it showed little improvement over the fourth-order one).

\footnotetext{
${ }^{12}$ However, it is worth noting that the form of the stochastic volatility process in Rudebusch and Swanson (2012) and Andreasen (2012) is different from the one specified here. In their case, it is possible that a fourth-order approximation would be sufficient to capture the standard deviation parameter of the stochastic volatility process in the solution.
} 
Figure 2: Accuracy of approximated solutions
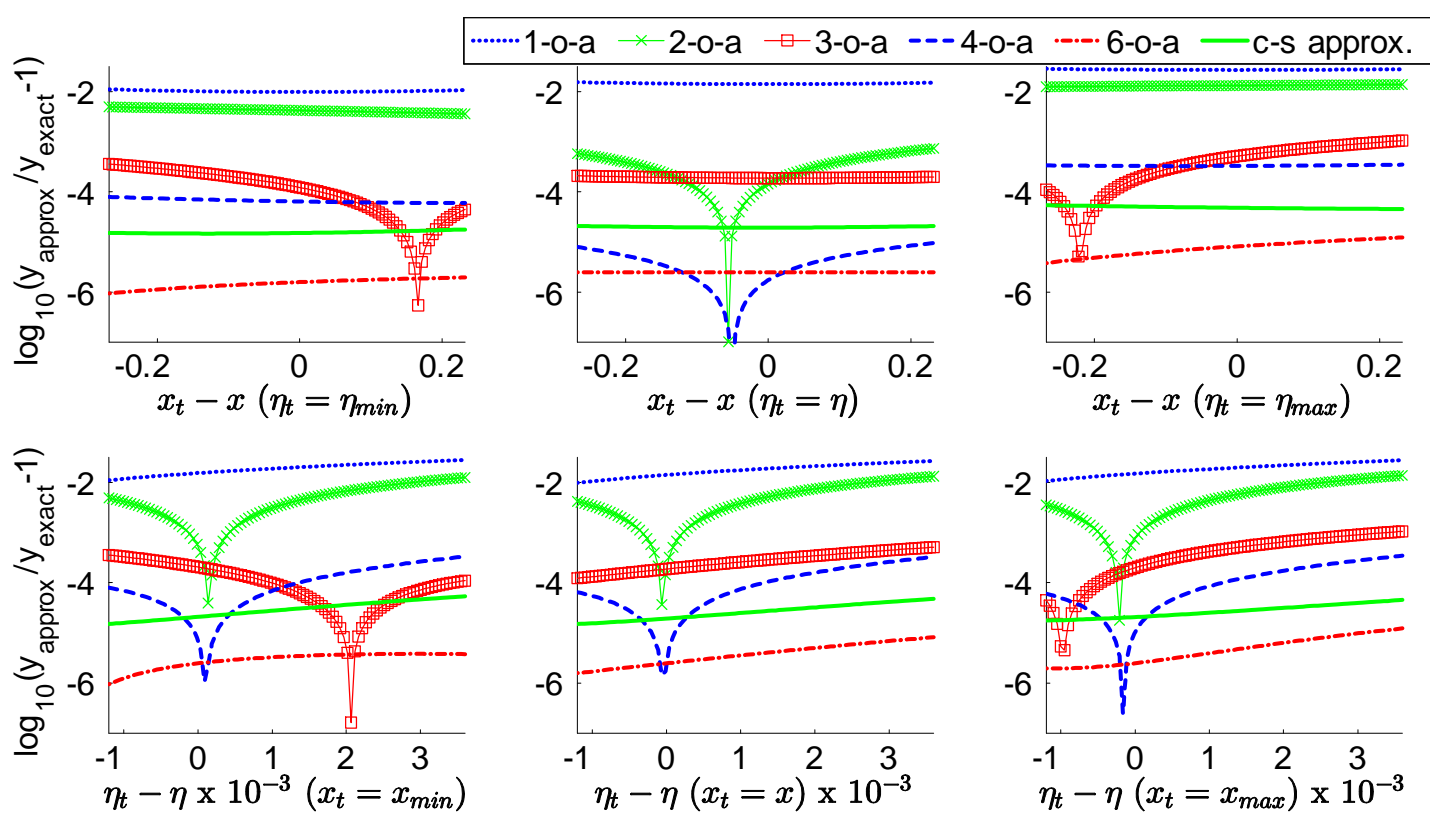

Note: 1-o-a to 6-o-a denotes the first- to sixth-order approximations in the neighbourhood of the deterministic steady state using perturbation methods. c-s approx. denotes the approximation utilized first by Campbell and Shiller, exploiting the normality of the stochastic processes.

$$
\eta_{\min }=0, \eta_{\max }=4 \eta, x_{\min }=-0.25, x_{\max }=0.25 \text {. }
$$




\section{Conclusion}

This paper provides an exact expression for the price-dividend ratio in an endowment asset pricing model with CRRA preferences, Gaussian autoregressive shocks, and stochastic volatility with innovations from any distribution for which the moment generating function exists. The solution provides a useful benchmark against which to test the performance of alternative numerical solution algorithms that one may wish to use to solve more elaborate macro-finance models with stochastic volatility. In particular, I show that perturbation methods may have to go higher than third order in order to fully capture the implications of stochastic volatility.

Since the structure of the model with stochastic volatility shares many of the properties of the basic Burnside asset pricing model, it should be possible to derive an exact solution for this stochastic volatility model with the addition of multivariate and higher-order autoregressive processes, as in Burnside (1998), or with habits in consumption, as in Chen et al. (2008) and Collard et al. (2006). This topic would be a fruitful direction for future research, as would a more thorough investigation of modelling stochastic volatility with the gamma distribution.

Acknowledgements I would like to thank the editor, C. Otrok, two anonymous referees, E. de Groot, M. Gonzalez-Astudillo, C. Karlsten and J. Roberts for useful discussions, insightful comments and careful proof reading.

\section{References}

Andreasen, M. M. (2012). On the effects of rare disasters and uncertainty shocks for risk premia in non-linear DSGE models. Review of Economic Dynamics 15(3), 295 -316 .

Bansal, R. and A. Yaron (2004). Risks for the long run: A potential resolution of asset pricing puzzles. The Journal of Finance 59(4), 1481-1509.

Bidarkota, P. V. and J. McCulloch (2003). Consumption asset pricing with stable shocks - exploring a solution and its implications for mean equity returns. Journal of Economic Dynamics and Control 27(3), 399 - 421.

Bloom, N., S. Bond, and J. van Reenen (2007). Uncertainty and investment dynamics. The Review of Economic Studies 74(2), pp. 391-415.

Burnside, C. (1998). Solving asset pricing models with gaussian shocks. Journal of Economic Dynamics and Control 22(3), 329 - 340.

Caldara, D., J. Fernández-Villaverde, J. F. Rubio-Ramírez, and W. Yao (2012). Computing DSGE models with recursive preferences and stochastic volatility. Review of 
Economic Dynamics 15(2), 188 - 206.

Calin, O. L., Y. Chen, T. F. Cosimano, and A. A. Himonas (2005). Solving asset pricing models when the price-dividend function is analytic. Econometrica 73(3), 961-982.

Campbell, J. and R. Shiller (1988). The dividend-price ratio and expectations of future dividends and discount factors. Review of Financial Studies 1(3), 195-228.

Chen, Y., T. Cosimano, and A. Himonas (2008). Solving an asset pricing model with hybrid internal and external habits, and autocorrelated Gaussian shocks. Annals of Finance 4(3), 305-344.

Collard, F., P. Féve, and I. Ghattassi (2006). A note on the exact solution of asset pricing models with habit persistence. Macroeconomic Dynamics 10, 273-283.

Fernández-Villaverde, J., P. A. Guerrón-Quintana, K. Kuester, and J. Rubio-Ramírez (2011). Fiscal volatility shocks and economic activity. Unpublished manuscript.

Gallant, A., D. Hsieh, and G. Tauchen (1997). Estimation of stochastic volatility models with diagnostics. Journal of Econometrics 81(1), 159 - 192.

Geweke, J. (1994). Bayesian analysis of stochastic volatility models: Comment. Journal of Business \& Economic Statistics 12(4), pp. 397-399.

Justiniano, A. and G. E. Primiceri (2008). The time-varying volatility of macroeconomic fluctuations. American Economic Review 98(3), 604-41.

Labadie, P. (1989). Stochastic inflation and the equity premium. Journal of Monetary Economics 24(2), $277-298$.

Le, A., K. J. Singleton, and Q. Dai (2010). Discrete-time affineQ term structure models with generalized market prices of risk. Review of Financial Studies 23(5), 21842227 .

Lucas, Robert E., J. (1978). Asset prices in an exchange economy. Econometrica 46(6), pp. 1429-1445.

Mehra, R. and E. C. Prescott (1985). The equity premium: A puzzle. Journal of Monetary Economics 15(2), 145 - 161.

Rudebusch, G. D. and E. T. Swanson (2012). The bond premium in a DSGE model with long-run real and nominal risks. American Economic Journal: Macroeconomics 4 (1), 105-43.

Schmitt-Grohé, S. and M. Uribe (2004). Solving dynamic general equilibrium models using a second-order approximation to the policy function. Journal of Economic Dynamics and Control 28(4), 755-775.

Sims, C. A. and T. Zha (2006). Were there regime switches in U.S. monetary policy? American Economic Review 96(1), 54-81. 
Stock, J. H. and M. W. Watson (2002). Has the business cycle changed and why? NBER Macroeconomics Annual 2002, Volume 17, 159-230.

Tsionas, E. G. (2003). Exact solution of asset pricing models with arbitrary shock distributions. Journal of Economic Dynamics and Control 27(5), $843-851$.

Weil, P. (1989). The equity premium puzzle and the risk-free rate puzzle. Journal of Monetary Economics 24(3), 401 - 421.

\section{A Appendix}

\section{A.1 The solution: Proof of Theorem 1}

The ultimate aim is to rewrite the expression

$$
\mathbf{E}_{t} \exp \left((1-\gamma) \sum_{j=1}^{i} x_{t+j}\right) \text { for } i=1,2, \ldots
$$

in terms of the time $t$ state variables, $x_{t}$ and $\eta_{t}$. Iterating forward the dividend growth process, equation (3), so that $x_{t+j}$ is in terms of $x_{t}$ gives

$$
x_{t+j}=x+\rho^{j}\left(x_{t}-x\right)+\sum_{k=1}^{j} \rho^{j-k} \sqrt{\eta_{t+k}} \varepsilon_{t+k} .
$$

Substituting this into (A.1) gives

$$
\mathbf{E}_{t} \exp \left((1-\gamma) \sum_{j=1}^{i}\left(x+\rho^{j}\left(x_{t}-x\right)+\sum_{k=1}^{j} \rho^{j-k} \sqrt{\eta_{t+k}} \varepsilon_{t+k}\right)\right) .
$$

Collecting terms for $x,\left(x_{t}-x\right)$ and each $\varepsilon_{t+j}$ gives

$$
\mathbf{E}_{t} \exp \left((1-\gamma)\left(\begin{array}{c}
\sum_{j=1}^{i}\left(x+\rho^{j}\left(x_{t}-x\right)\right) \\
+\sum_{j=1}^{i}\left(\sum_{k=1}^{i-j+1} \rho^{k-1}\right) \sqrt{\eta_{t+j}} \varepsilon_{t+j}
\end{array}\right)\right)
$$

Using the standard results of geometric progressions gives

$$
\mathbf{E}_{t} \exp \left(\begin{array}{c}
(1-\gamma) i x+(1-\gamma) \rho \frac{1-\rho^{i}}{1-\rho}\left(x_{t}-x\right) \\
+\frac{(1-\gamma)}{1-\rho} \sum_{j=1}^{i}\left(1-\rho^{i-j+1}\right) \sqrt{\eta_{t+j}} \varepsilon_{t+j}
\end{array}\right)
$$

Since the first row in the previous expression is only in terms of $x$ and $\left(x_{t}-x\right)$, the expectations operator can be moved, leaving

$$
\exp \left(A_{i} x+B_{i}\left(x_{t}-x\right)\right) \mathbf{E}_{t} \exp \left(\theta \sum_{j=1}^{i}\left(1-\rho^{i-j+1}\right) \sqrt{\eta_{t+j}} \varepsilon_{t+j}\right)
$$

where

$$
A_{i} \equiv(1-\gamma) i, \quad B_{i} \equiv \theta \rho\left(1-\rho^{i}\right) \quad \text { and } \quad \theta \equiv\left(\frac{1-\gamma}{1-\rho}\right)
$$


At this stage it is instructive to rewrite the expression with the expectations operator in (A.2) as an integral of probabilistic outcomes

$$
\underset{\varepsilon_{\eta, t+1}}{\int} \cdots \int_{\varepsilon_{\eta, t+i} \varepsilon_{t+1}} \cdots \int_{\varepsilon_{t+i}} \exp \left(\theta \sum_{j=1}^{i}\left(1-\rho^{i-j+1}\right) \sqrt{\eta_{t+j}} \varepsilon_{t+j}\right) \underset{\varepsilon_{t+1}}{d F} \cdots \underbrace{}_{\varepsilon_{t+i} \varepsilon_{\eta, t+1}} d F_{\eta} \cdots d F_{\varepsilon_{\eta, t+i}}
$$

where $F$ and $F_{\eta}$ are the density functions for the i.i.d. random variables $\varepsilon$ and $\varepsilon_{\eta}$, respectively. Since the $\varepsilon$ innovations are independent, we can rewrite the problem as

$$
\underset{\varepsilon_{\eta, t+1}}{\int \cdots \int_{\varepsilon_{\eta, t+i}}}\left(\prod_{j=1}^{i} \int_{\varepsilon_{t+j}} \exp \left(\theta\left(1-\rho^{i-j+1}\right) \sqrt{\eta_{t+j}} \varepsilon_{t+j}\right) \underset{\varepsilon_{t+j}}{d F}\right) \underset{\varepsilon_{\eta, t+1}}{d F_{\eta}} \cdots \underset{\varepsilon_{\eta, t+i}}{d F_{\eta}}
$$

Using a standard result for random variables, namely that if $z \sim N(0,1)$ and $k$ is a scalar, then $\mathbf{E}(\exp (k z))=\exp \left(\frac{k^{2}}{2}\right)$, we get

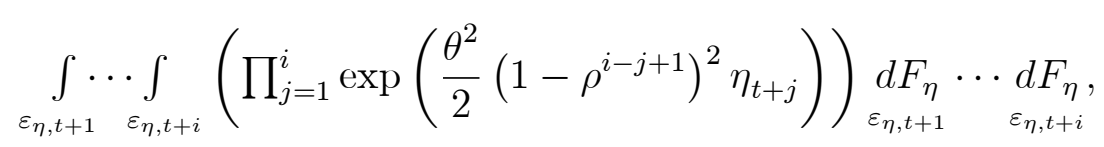

or

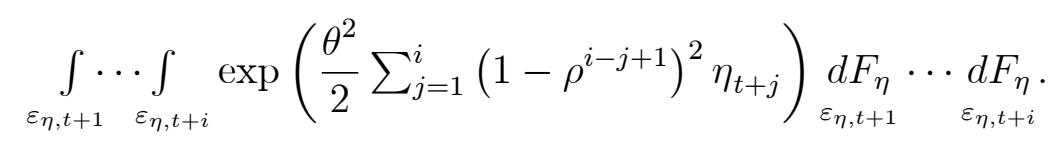

If we assumed $\eta_{t+i}=\eta$ for all $i=1,2, \ldots$ the expectations operator would disappear from the above expression and with a little further manipulation we would recover the solution in Burnside (1998). Instead, with stochastic volatility there is more work to do. Iterating forward the stochastic volatility process, equation (4), so that $\eta_{t+j}$ is in terms of $\eta_{t}$ gives

$$
\eta_{t+j}=\eta+\rho_{\eta}^{j}\left(\eta_{t}-\eta\right)+\sum_{k=1}^{j} \rho_{\eta}^{j-k} \omega \varepsilon_{\eta, t+k}
$$

Substituting this expression into (A.3) gives

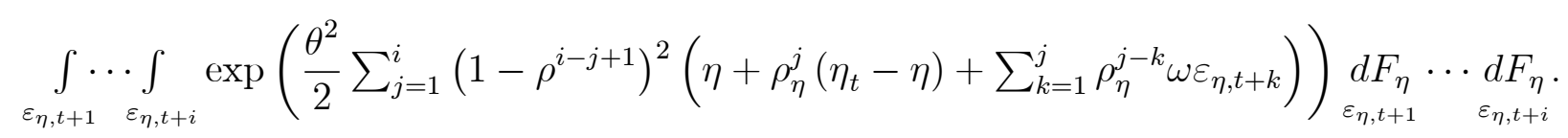

Collecting terms for $\eta,\left(\eta_{t}-\eta\right)$ and each $\varepsilon_{\eta, t+j}$ gives

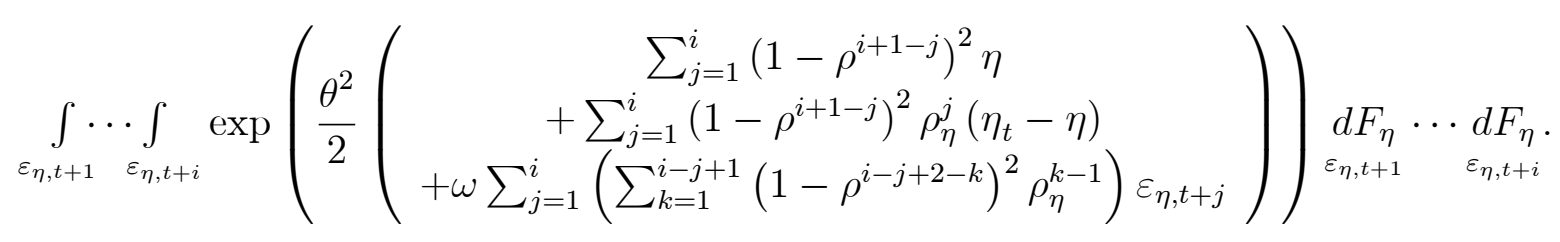


Since the first two rows in the previous expression are only in terms of $\eta$ and $\left(\eta_{t}-\eta\right)$, the integral can be moved, leaving

$$
\begin{aligned}
& \exp \left(C_{i} \eta+D_{i}\left(\eta_{t}-\eta\right)\right) \\
& \times \int_{\varepsilon_{\eta, t+1}} \cdots \int_{\varepsilon_{\eta, t+i}} \exp \left(\frac{\theta^{2} \omega}{2} \sum_{j=1}^{i}\left(\sum_{k=1}^{i-j+1}\left(1-\rho^{i-j+2-k}\right)^{2} \rho_{\eta}^{k-1}\right) \varepsilon_{\eta, t+j}\right) \underset{\varepsilon_{\eta, t+1}}{d F_{\eta}} \cdots d F_{\varepsilon_{\eta, t+i}},
\end{aligned}
$$

where

$$
C_{i} \equiv \frac{\theta^{2}}{2} \sum_{j=1}^{i}\left(1-\rho^{i+1-j}\right)^{2} \quad \text { and } \quad D_{i} \equiv \frac{\theta^{2}}{2} \sum_{j=1}^{i}\left(1-\rho^{i+1-j}\right)^{2} \rho_{\eta}^{j} .
$$

Notice that $D_{i} \geq 0, \frac{\partial D_{i}}{\partial \rho} \leq 0$ and $\frac{\partial D_{i}}{\partial \rho_{\eta}} \geq 0$. Expanding the quadratic terms in $C_{i}$ and $D_{i}$ gives

$$
\begin{gathered}
C_{i}=\frac{\theta^{2}}{2} \sum_{j=1}^{i}\left(1-2 \rho^{i} \rho^{-(j-1)}+\rho^{2 i} \rho^{-2(j-1)}\right), \\
D_{i}=\frac{\theta^{2}}{2} \sum_{j=1}^{i}\left(\rho_{\eta} \rho_{\eta}^{j-1}-2 \rho_{\eta} \rho^{i}\left(\rho_{\eta} \rho^{-1}\right)^{j-1}+\rho_{\eta} \rho^{2 i}\left(\rho_{\eta} \rho^{-2}\right)^{j-1}\right),
\end{gathered}
$$

and using the standard results of geometric progressions gives

$$
\begin{gathered}
C_{i}=\frac{\theta^{2}}{2}\left(i-2 \rho \frac{1-\rho^{i}}{1-\rho}+\rho^{2} \frac{1-\rho^{2 i}}{1-\rho^{2}}\right) \\
D_{i}=\frac{\theta^{2}}{2}\left(\rho_{\eta} \frac{1-\rho_{\eta}^{i}}{1-\rho_{\eta}}-2 \rho_{\eta}^{i} \rho \frac{1-\left(\rho_{\eta}^{-1} \rho\right)^{i}}{1-\rho_{\eta}^{-1} \rho}+\rho_{\eta}^{i} \rho^{2} \frac{1-\left(\rho_{\eta}^{-1} \rho^{2}\right)^{i}}{1-\rho_{\eta}^{-1} \rho^{2}}\right)
\end{gathered}
$$

Collecting terms in $D_{i}$ gives

$$
D_{i}=\frac{\theta^{2} \rho_{\eta}}{2}\left(\phi_{1}+\phi_{2} \rho_{\eta} \rho_{\eta}^{i-1}+\phi_{3} \rho^{i-1}+\phi_{4} \rho^{2(i-1)}\right)
$$

where

$$
\phi_{1} \equiv \frac{1}{1-\rho_{\eta}}, \quad \phi_{2} \equiv \frac{-\rho_{\eta}\left(\rho_{\eta}+\rho\right)(1-\rho)^{2}}{\left(\rho^{2}-\rho_{\eta}\right)\left(\rho-\rho_{\eta}\right)\left(1-\rho_{\eta}\right)}, \quad \phi_{3} \equiv \frac{-2 \rho^{2}}{\rho-\rho_{\eta}}, \quad \text { and } \quad \phi_{4} \equiv \frac{\rho^{4}}{\rho^{2}-\rho_{\eta}} .
$$

The final expression left to evaluate is the integral expression in (A.4),

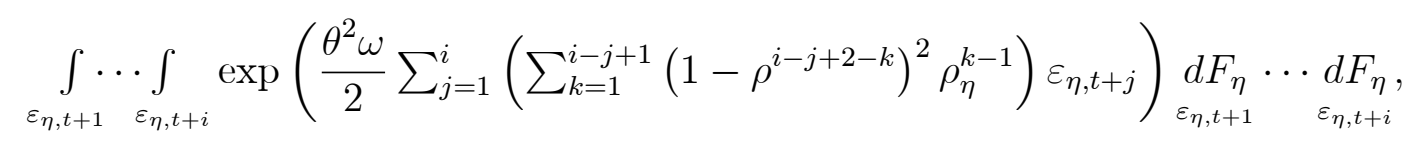

which can be rewritten as

$$
\prod_{j=1}^{i} \int_{\varepsilon_{\eta, t+j}} \exp \left(\frac{\theta^{2} \omega}{2}\left(\sum_{k=1}^{i-j+1}\left(1-\rho^{i-j+2-k}\right)^{2} \rho_{\eta}^{k-1}\right) \varepsilon_{\eta, t+j}\right) \underset{\varepsilon_{\eta, t+j}}{d F_{\eta}} .
$$

Let the moment generating function $(\mathrm{MGF})$ for the i.i.d. random variable $\varepsilon_{\eta}$ be

$$
M(\tau)=\mathbf{E} \exp \left(\tau \varepsilon_{\eta}\right)=\int_{-\infty}^{\infty} \exp \left(\tau \varepsilon_{\eta}\right) d F_{\eta}\left(\varepsilon_{\eta}\right), \quad \tau \in \mathbf{R}
$$


Rewriting expression (A.7) using the MGF notation gives

$$
\prod_{j=1}^{i} M\left(\tau_{j}\right)
$$

where

$$
\tau_{j}=\frac{\theta^{2} \omega}{2}\left(\sum_{k=1}^{i-j+1}\left(1-\rho^{i-j+2-k}\right)^{2} \rho_{\eta}^{k-1}\right)
$$

Using the results of geometric progressions, $\sum_{k=1}^{i-j+1}\left(1-\rho^{i-j+2-k}\right)^{2} \rho_{\eta}^{k-1}$ can be rewritten as

$$
\phi_{1}+\phi_{2} \rho_{\eta} \rho_{\eta}^{i-j}+\phi_{3} \rho^{i-j}+\phi_{4} \rho^{2(i-j)} .
$$

The term $H_{i}$ in equation (8) from the main text is therefore given by

$$
\sum_{j=1}^{i} \log M\left(\frac{\theta^{2} \omega}{2}\left(\phi_{1}+\phi_{2} \rho_{\eta} \rho_{\eta}^{i-j}+\phi_{3} \rho^{i-j}+\phi_{4} \rho^{2(i-j)}\right)\right) .
$$

This completes the proof.

\section{A.2 The standard normal distribution: Proof of Corollary 2}

The MGF of the standard normal distribution is

$$
M^{S t N}(\tau)=\exp \left(\frac{\tau^{2}}{2}\right)
$$

Applying (A.9) to $H_{i}$ in equation (8) gives $F_{i} \omega^{2}$ where

$$
F_{i}=\frac{\theta^{4}}{8} \sum_{j=1}^{i}\left(\phi_{1}+\phi_{2} \rho_{\eta} \rho_{\eta}^{i-j}+\phi_{3} \rho^{i-j}+\phi_{4} \rho^{2(i-j)}\right)^{2}
$$

It is also possible to apply the standard normal MGF to (A.7), which gives

$$
F_{i}=\frac{\theta^{4}}{8} \sum_{j=1}^{i}\left(\sum_{k=1}^{i-j+1}\left(1-\rho^{i-j+2-k}\right)^{2} \rho_{\eta}^{k-1}\right)^{2} .
$$

and makes it clear that $F_{i} \geq 0, \frac{\partial F_{i}}{\partial \rho} \leq 0$ and $\frac{\partial F_{i}}{\partial \rho_{\eta}} \geq 0$. Equation (A.10) is another geometric progression (albeit a more tedious one). It is useful to reverse the indexation for $j=1, \ldots, i$ by rewriting $i-j$ as $j-1$, in which case

$$
F_{i}=\frac{\theta^{4}}{8} \sum_{j=1}^{i}\left(\phi_{1}+\phi_{2} \rho_{\eta}^{j-1}+\phi_{3} \rho^{j-1}+\phi_{4} \rho^{2(j-1)}\right)^{2} .
$$


Multiplying out the quadratic term gives

$$
F_{i}=\frac{\theta^{4}}{8} \sum_{j=1}^{i}\left(\begin{array}{c}
\phi_{1}^{2}+\phi_{2}^{2} \rho_{\eta}^{2(j-1)}+\phi_{3}^{2} \rho^{2(j-1)}+\phi_{4}^{2} \rho^{4(j-1)} \\
+2 \phi_{1} \phi_{2} \rho_{\eta}^{j-1}+2 \phi_{1} \phi_{3} \rho^{j-1}+2 \phi_{1} \phi_{4} \rho^{2(j-1)} \\
+2 \phi_{2} \phi_{3}\left(\rho_{\eta} \rho\right)^{j-1}+2 \phi_{2} \phi_{4}\left(\rho_{\eta} \rho^{2}\right)^{j-1}+2 \phi_{3} \phi_{4} \rho^{3(j-1)}
\end{array}\right)
$$

Using (for the final time) the results of geometric progressions gives

$$
F_{i}=\frac{\theta^{4}}{8}\left(\begin{array}{c}
i \phi_{1}^{2}+\phi_{2}^{2} \frac{1-\rho_{\eta}^{2 i}}{1-\rho_{\eta}^{2}}+\phi_{3}^{2} \frac{1-\rho^{2 i}}{1-\rho^{2}}+\phi_{4}^{2} \frac{1-\rho^{4 i}}{1-\rho^{4}} \\
+2 \phi_{1} \phi_{2} \frac{1-\rho_{\eta}^{i}}{1-\rho_{\eta}}+2 \phi_{1} \phi_{3} \frac{1-\rho^{i}}{1-\rho}+2 \phi_{1} \phi_{4} \frac{1-\rho^{2 i}}{1-\rho^{2}} \\
+2 \phi_{2} \phi_{3} \frac{1-\left(\rho_{\eta} \rho\right)^{i}}{1-\rho_{\eta} \rho}+2 \phi_{2} \phi_{4} \frac{1-\left(\rho_{\eta} \rho^{2}\right)^{i}}{1-\rho_{\eta} \rho^{2}}+2 \phi_{3} \phi_{4} \frac{1-\rho^{3 i}}{1-\rho^{3}}
\end{array}\right)
$$

This completes the proof.

\section{A.3 Existence: Proof of Theorem 3}

The aim is to show that the infinite summation

$$
\sum_{i=1}^{\infty} \beta^{i} \exp \left(A_{i} x+B_{i}\left(x_{t}-x\right)+C_{i} \eta+D_{i}\left(\eta_{t}-\eta\right)+F_{i} \omega^{2}\right)
$$

convergences to a finite number. First, I define

$$
z_{i} \equiv \beta^{i} \exp \left(A_{i} x+B_{i}\left(x_{t}-x\right)+C_{i} \eta+D_{i}\left(\eta_{t}-\eta\right)+F_{i} \omega^{2}\right),
$$

so that the price-dividend ratio given by $y_{t}=\sum_{i=1}^{\infty} z_{i}$. To test convergence, it is sufficient to show that $\lim _{i \rightarrow \infty}\left|\frac{z_{i+1}}{z_{i}}\right|<1$. It follows that

$$
\left|\frac{z_{i+1}}{z_{i}}\right|=\beta \exp \left(\widetilde{A} x+\widetilde{B}_{i}\left(x_{t}-x\right)+\widetilde{C}_{i} \eta+\widetilde{D}_{i}\left(\eta_{t}-\eta\right)+\widetilde{F}_{i} \omega^{2}\right)
$$

where the notation, $\widetilde{X_{i}} \equiv X_{i+1}-X_{i}$ is used, and where

$$
\begin{gathered}
\widetilde{A} \equiv 1-\gamma, \quad \widetilde{B}_{i} \equiv(1-\gamma) \rho^{i+1} \\
\widetilde{C}_{i} \equiv \frac{\theta^{2}}{2}\left(1-2 \rho^{i+1}+\rho^{2(i+1)}\right) \\
\widetilde{D}_{i} \equiv \frac{\theta^{2} \rho_{\eta}}{2}\left(\phi_{2} \rho_{\eta}\left(1-\rho_{\eta}^{-1}\right) \rho_{\eta}^{i}+\phi_{3} \rho^{i}\left(1-\rho^{-1}\right)+\phi_{4} \rho^{2 i}\left(1-\rho^{-2}\right)\right) \\
\text { and } \widetilde{F}_{i} \equiv \frac{\theta^{4}}{8}\left(\begin{array}{c}
\phi_{1}^{2}+\phi_{2}^{2} \rho_{\eta}^{2 i}+\phi_{3}^{2} \rho^{2 i}+\phi_{4}^{2} \rho^{4 i} \\
+2 \phi_{1} \phi_{2} \rho_{\eta}^{i}+2 \phi_{1} \phi_{3} \rho^{i}+2 \phi_{1} \phi_{4} \rho^{2 i} \\
+2 \phi_{2} \phi_{3}\left(\rho_{\eta} \rho\right)^{i}+2 \phi_{2} \phi_{4}\left(\rho_{\eta} \rho^{2}\right)^{i}+2 \phi_{3} \phi_{4} \rho^{3 i}
\end{array}\right)
\end{gathered}
$$


Taking the limit of these terms gives

$$
\begin{gathered}
\lim _{i \rightarrow \infty} \widetilde{A}=1-\gamma, \quad \lim _{i \rightarrow \infty} \widetilde{B}_{i}=0 \\
\lim _{i \rightarrow \infty} \widetilde{C}_{i}=\frac{1}{2}\left(\frac{1-\gamma}{1-\rho}\right)^{2}, \\
\lim _{i \rightarrow \infty} \widetilde{D}_{i}=0, \text { and } \lim _{i \rightarrow \infty} \widetilde{F}_{i}=\frac{\theta^{4}}{8\left(1-\rho_{\eta}\right)^{2}} .
\end{gathered}
$$

It then follows that

$$
\lim _{i \rightarrow \infty}\left|\frac{z_{i+1}}{z_{i}}\right|=\beta \exp \left((1-\gamma) x+\frac{1}{2}\left(\frac{1-\gamma}{1-\rho}\right)^{2} \eta+\frac{\theta^{4}}{8\left(1-\rho_{\eta}\right)^{2}} \omega^{2}\right) .
$$

This completes the proof.

\section{B Online appendix (not for publication)}

\section{B.1 Alternative moment generating functions}

This section applies and discusses two alternative distributions for $\varepsilon_{\eta}$ for which the MGF exists: The truncated normal and gamma distribution, respectively.

\section{B.1.1 Non-negative volatility with the truncated normal distribution}

Drawing the $\varepsilon_{\eta}$ innovations from the standard normal distribution creates the technical possibility that we get negative values for $\eta_{t}$. One solution to this problem is to draw from a truncated standard normal distribution which, with appropriate truncation, can guarantee non-negative values for $\eta_{t}$. To find the natural truncation point, calculate the value of $\eta_{t+i}$ (without loss of generality, we set $\eta_{t}=\eta$ ) following a sequence of lowestpossible realizations of $\varepsilon_{\eta}$, namely $\varepsilon_{\eta}^{\min }$ to give

$$
\eta_{t+i}^{\min }=\eta+\rho_{\eta}^{i-1} \omega \varepsilon_{\eta}^{\min }+\cdots+\omega \varepsilon_{\eta}^{\min } .
$$

The non-negativity constraint requires $\lim _{i \rightarrow \infty} \eta_{t+i}^{\min }>0$, in which case

$$
\eta+\lim _{i \rightarrow \infty} \frac{1-\rho_{\eta}^{i}}{1-\rho_{\eta}} \omega \varepsilon_{\eta}^{\min }>0 \quad \text { or } \quad \varepsilon_{\eta}^{\min }>-\frac{\eta\left(1-\rho_{\eta}\right)}{\omega} .
$$

This expression implies that for a small $\omega$ relative to a large $\eta$ (and low persistence, $\rho_{\eta}$ ), the probability of $\eta_{t}$ becoming negative can be small and of no practical concern. Bansal and Yaron (2004) use the following parameterization for the stochastic volatility process: $\eta=6.08 \times 10^{-5}, \rho_{\eta}=0.987$, and $\omega=0.23 \times 10^{-5}$. In this case $\varepsilon_{\eta}^{\min }=-0.344$. However, drawing from this distribution would also lower the volatility of the process that Bansal 
and Yaron (2004) targeted since

$$
\operatorname{var}\left(\varepsilon_{\eta}^{T r N}\right)=1+\frac{2 \varepsilon_{\eta}^{\min } \phi\left(\varepsilon_{\eta}^{\min }\right)}{1-2 \Phi\left(\varepsilon_{\eta}^{\min }\right)}<1
$$

where the $\operatorname{Tr} N$ superscript denotes that it is the truncated random variable and the 1 on the right-hand side of the expression is the variance of the non-truncated standard normal. With $\varepsilon_{\eta}$ drawn from a symmetrically truncated standard normal distribution with $\varepsilon_{\eta}^{\min }=-\frac{\eta\left(1-\rho_{\eta}\right)}{\omega}$, the MGF is given by

$$
M^{T r N}(\tau)=\exp \left(\frac{\tau^{2}}{2}\right)\left(\frac{\Phi\left(-\varepsilon_{\eta}^{\min }-\tau\right)-\Phi\left(\varepsilon_{\eta}^{\min }-\tau\right)}{1-2 \Phi\left(\varepsilon_{\eta}^{\min }\right)}\right) .
$$

In the limit, $\frac{\eta\left(1-\rho_{\eta}\right)}{\omega} \rightarrow \infty$, the moment generating function would be $\exp \left(\frac{\tau^{2}}{2}\right)$, recovering the solution for the standard normal distribution.

\section{B.1.2 Fat tails with the gamma distribution}

Another possible solution to the above problem of non-negativity is the gamma distribution since the support is $\varepsilon_{\eta}^{\Gamma} \in(0, \infty)$. This gamma distribution's MGF is given by

$$
M^{\Gamma}(\tau)=\left(1-g_{1} \tau\right)^{-g_{2}} \quad \text { for } \quad \tau<\frac{1}{g_{1}} .
$$

An additional benefit of the gamma distribution is that it generates both positive skew, $\frac{2}{\sqrt{g_{2}}}$ and excess kurtosis, $\frac{6}{g_{2}}$. Suppose that the shape parameter, $g_{2}$ is used to match some moment in the data that captures skewness or kurtosis. The scale parameter, $g_{1}$ can then be calibrated as follows: The gamma distribution has the following properties

$$
\mathbf{E}\left(\varepsilon_{\eta}^{\Gamma}\right)=g_{1} g_{2} \quad \text { and } \quad \mathbf{V}\left(\varepsilon_{\eta}^{\Gamma}\right)=g_{1}^{2} g_{2}
$$

Assume that the parameter pair $\left(\eta^{S t N}, \omega^{S t N}\right)$ were calibrated to match $\mathbf{E}\left(\eta_{t}\right)$ and $\mathbf{V}\left(\eta_{t}\right)$, the unconditional mean and variance of the stochastic volatility process, respectively, where $S t N$ denotes the calibration for the standard normal distribution. These formula for unconditional mean and variance is given by

$$
\mathbf{E}\left(\eta_{t}\right)=\eta+\frac{\omega}{\left(1-\rho_{\eta}\right)} \mathbf{E}\left(\varepsilon_{\eta}\right) \quad \text { and } \quad \mathbf{V}\left(\eta_{t}\right)=\frac{\omega^{2}}{\left(1-\rho_{\eta}^{2}\right)} \mathbf{V}\left(\varepsilon_{\eta}\right)
$$

We therefore have the following two expressions

$$
\eta^{S t N}=\eta^{\Gamma}+\frac{\omega^{\Gamma}}{\left(1-\rho_{\eta}\right)} g_{1} g_{2} \quad \text { and } \quad\left(\omega^{S t N}\right)^{2}=\left(\omega^{\Gamma}\right)^{2} g_{1}^{2} g_{2}
$$


Normalizing $\omega^{\Gamma}=\omega^{S t N}$, we have the restriction that $g_{1}=\frac{1}{\sqrt{g_{2}}}$. Substituting this into the first equation determines the appropriate value for $\eta$ :

$$
\eta^{\Gamma}=\eta^{S t N}-\frac{\omega^{\Gamma}}{\left(1-\rho_{\eta}\right)} g_{1} g_{2} .
$$

In the online Appendix B.5, the model is solved with both a standard normal and gamma distribution under a given calibration and the price-dividend ratio and risk-free rate are reported.

\section{B.2 Additional variables}

This section derives closed-form solutions for several additional variables of interest, including the mean price-dividend ratio, the risk-free rate and the conditional-expected equity return and conditional-expected equity risk premium.

\section{B.2.1 Unconditional mean price-dividend ratio}

In order to calculate the unconditional mean, it is necessary to appropriately capture the autocorrelation created by the $\varepsilon_{\eta}$ innovations in the dividend growth process. Iterating backward the stochastic volatility process, equation (4), so that $\eta_{t}$ is in terms of a sequence of past $\varepsilon_{\eta}$ realizations gives

$$
\eta_{t}-\eta=\rho_{\eta}^{k}\left(\eta_{t-k}-\eta\right)+\omega \sum_{s=1}^{k} \rho_{\eta}^{s-1} \varepsilon_{\eta, t+1-s}
$$

Taking the limit gives

$$
\lim _{k \rightarrow \infty} \eta_{t}-\eta=\omega \sum_{s=1}^{\infty} \rho_{\eta}^{s-1} \varepsilon_{\eta, t+1-s}
$$

in which case

$$
\eta_{t+1-j}-\eta=\omega \sum_{s=1}^{\infty} \rho_{\eta}^{s-1} \varepsilon_{\eta, t+2-j-s} .
$$

Similarly, $x_{t}$ can be written as

$$
x_{t}-x=\rho^{k}\left(x_{t-k}-x\right)+\sum_{j=1}^{k} \rho^{j-1} \sqrt{\eta_{t+1-j}} \varepsilon_{t+1-j},
$$

and

$$
\lim _{k \rightarrow \infty} x_{t}-x=\sum_{j=1}^{\infty} \rho^{j-1} \sqrt{\eta_{t+1-j}} \varepsilon_{t+1-j} .
$$

Substituting in for equation (B.1) gives

$$
x_{t}-x=\sum_{j=1}^{\infty} \rho^{j-1}\left(\sqrt{\eta+\omega \sum_{s=1}^{\infty} \rho_{\eta}^{s-1} \varepsilon_{\eta, t+2-j-s}}\right) \varepsilon_{t+1-j} .
$$


The unconditional mean of $y_{t}$ is

$$
\mathbf{E}\left(y_{t}\right)=\sum_{i=1}^{\infty} \beta^{i} \exp \left(Z_{i}\right) \mathbf{E} \exp \left(B_{i}\left(x_{t}-x\right)+D_{i}\left(\eta_{t}-\eta\right)\right)
$$

where

$$
Z_{i} \equiv A_{i} x+C_{i} \eta+F_{i} \omega^{2}
$$

which means we need only evaluate the expectations term

$$
\mathbf{E} \exp \left(B_{i}\left(x_{t}-x\right)+D_{i}\left(\eta_{t}-\eta\right)\right)
$$

To do this, first substitute using equation (B.2), which gives

$\mathbf{E} \exp \left(B_{i}\left(\sum_{j=1}^{\infty} \rho^{j-1}\left(\sqrt{\eta+\omega \sum_{s=1}^{\infty} \rho_{\eta}^{s-1} \varepsilon_{\eta, t+2-j-s}}\right) \varepsilon_{t+1-j}\right)+D_{i}\left(\omega \sum_{j=1}^{\infty} \rho_{\eta}^{j-1} \varepsilon_{\eta, t+1-j}\right)\right)$.

At this stage it is instructive to rewrite the expectations operator as an integral of probabilistic outcomes

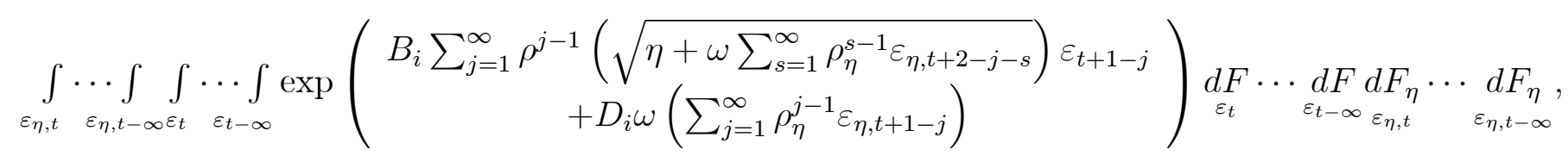

Rearranging the above expression gives

$$
\begin{aligned}
& \underset{\varepsilon_{\eta, t}}{\int} \cdots \int_{\varepsilon_{\eta, t-\infty}}\left(\prod_{j=1}^{\infty} \int_{\varepsilon_{t}} \cdots \int_{\varepsilon_{t-\infty}} \exp \left(B_{i} \rho^{j-1}\left(\sqrt{\eta+\omega \sum_{s=1}^{\infty} \rho_{\eta}^{s-1} \varepsilon_{\eta, t+2-j-s}}\right) \varepsilon_{t+1-j}\right) d F \cdots{ }_{\varepsilon_{t}} \cdots d F\right. \\
& \times \exp \left(D_{i} \omega\left(\sum_{j=1}^{\infty} \rho_{\eta}^{j-1} \varepsilon_{\eta, t+1-j}\right)\right) \underset{\varepsilon_{\eta, t}}{d F_{\eta} \cdots d F_{\eta, t-\infty}} .
\end{aligned}
$$

Using the same result as before for standard normal distributions gives

$$
\begin{aligned}
& \quad \underset{\varepsilon_{\eta, t}}{\int} \cdots \int_{\varepsilon_{\eta, t-\infty}} \prod_{j=1}^{\infty} \exp \left(\frac{B_{i}^{2}}{2} \rho^{2(j-1)}\left(\eta+\omega \sum_{s=1}^{\infty} \rho_{\eta}^{s-1} \varepsilon_{\eta, t+2-j-s}\right)\right) \\
& \times \exp \left(D_{i} \omega\left(\sum_{j=1}^{\infty} \rho_{\eta}^{j-1} \varepsilon_{\eta, t+1-j}\right)\right) \underset{\substack{d F_{\eta, t} \\
F_{\eta} \cdots}}{d F_{\eta, t-\infty}},
\end{aligned}
$$

which can be rewritten as

$$
\underset{\varepsilon_{\eta, t}}{\int} \cdots \int_{\varepsilon_{\eta, t-\infty}} \exp \left(\frac{B_{i}^{2}}{2} \sum_{j=1}^{\infty} \rho^{2(j-1)}\left(\eta+\omega \sum_{s=1}^{\infty} \rho_{\eta}^{s-1} \varepsilon_{\eta, t+2-j-s}\right)+D_{i} \omega\left(\sum_{j=1}^{\infty} \rho_{\eta}^{j-1} \varepsilon_{\eta, t+1-j}\right)\right) d F_{\eta} \cdots \underset{\varepsilon_{\eta, t}}{\cdots} d F_{\eta},
$$


Removing the constants term from the integral gives

$$
\exp \left(K_{i}\right) \int_{\varepsilon_{\eta, t}} \cdots \int_{\varepsilon_{\eta, t-\infty}} \exp \left(\begin{array}{c}
\frac{B_{i}^{2} \omega}{2} \sum_{j=1}^{\infty} \rho^{2(j-1)}\left(\sum_{s=1}^{\infty} \rho_{\eta}^{s-1} \varepsilon_{\eta, t+2-j-s}\right) \\
+D_{i} \omega\left(\sum_{j=1}^{\infty} \rho_{\eta}^{j-1} \varepsilon_{\eta, t+1-j}\right)
\end{array}\right) d F_{\eta} \cdots d F_{\eta},
$$

where

$$
K_{i} \equiv \frac{B_{i}^{2} \eta}{2\left(1-\rho^{2}\right)}
$$

Focussing on the integral term, the above expression is rearranged in order to bring together $\varepsilon_{\eta}$ innovations with the same time subscript:

$$
\underset{\varepsilon_{\eta, t}}{\int} \cdots \int_{\varepsilon_{\eta, t-\infty}} \exp \left(\sum_{j=1}^{\infty}\left(\frac{B_{i}^{2} \omega}{2} \rho_{\eta}^{j-1}\left(\sum_{s=1}^{j}\left(\rho_{\eta}^{-1} \rho^{2}\right)^{s-1}\right)+D_{i} \omega \rho_{\eta}^{j-1}\right) \varepsilon_{\eta, t+1-j}\right) \underset{\substack{\varepsilon_{\eta, t} \\ \varepsilon_{\eta}}}{d F_{\varepsilon_{\eta, t-\infty}}} d F_{\eta} .
$$

Again, using the results of standard normals and geometric series gives

$$
\exp \left(\frac{\omega^{2}}{2} \sum_{j=1}^{\infty}\left(\frac{B_{i}^{2}}{2} \rho_{\eta}^{j-1}\left(\frac{1-\left(\rho_{\eta}^{-1} \rho^{2}\right)^{j}}{1-\rho_{\eta}^{-1} \rho^{2}}\right)+D_{i} \rho_{\eta}^{j-1}\right)^{2}\right)
$$

This can be rewritten as

$$
\exp \left(\frac{\omega^{2}}{2} \sum_{j=1}^{\infty}\left(\gamma_{1} \rho_{\eta}^{j-1}-\gamma_{2} \rho^{2(j-1)}\right)^{2}\right)
$$

where

$$
\gamma_{i, 1} \equiv\left(\frac{B_{i}^{2}}{2} \frac{\rho_{\eta}}{\rho_{\eta}-\rho^{2}}+D_{i}\right) \text { and } \gamma_{i, 2} \equiv \frac{B_{i}^{2}}{2} \frac{\rho^{2}}{\rho_{\eta}-\rho^{2}}
$$

Multiplying out the quadratic term in expression (B.3) gives

$$
\exp \left(\frac{\omega^{2}}{2} \sum_{j=1}^{\infty}\left(\gamma_{i, 1}^{2} \rho_{\eta}^{2(j-1)}-2 \gamma_{i, 1} \gamma_{i, 2}\left(\rho_{\eta} \rho^{2}\right)^{j-1}+\gamma_{2}^{2} \rho^{4(j-1)}\right)\right)
$$

And using the standard results of geometric series gives

$$
\exp \left(\frac{\omega^{2}}{2}\left(\frac{\gamma_{1}^{2}}{1-\rho_{\eta}^{2}}-\frac{2 \gamma_{1} \gamma_{2}}{1-\rho_{\eta} \rho^{2}}+\frac{\gamma_{2}^{2}}{1-\rho^{4}}\right)\right)
$$

Thus, the unconditional mean price-dividend ratio is

$$
\mathbf{E} y_{t}=\sum_{i=1}^{\infty} \beta^{i} \exp \left(A_{i} x+\left(C_{i}+\frac{B_{i}^{2}}{2\left(1-\rho^{2}\right)}\right) \eta+\left(\frac{1}{2}\left(\frac{\gamma_{i, 1}^{2}}{1-\rho_{\eta}^{2}}-\frac{2 \gamma_{i, 1} \gamma_{i, 2}}{1-\rho_{\eta} \rho^{2}}+\frac{\gamma_{i, 2}^{2}}{1-\rho^{4}}\right)+F_{i}\right) \omega^{2}\right)
$$


Next, it is necessary to show that the condition for convergence of the infinite summation in the expression above is the same as the condition stated in Theorem 3. Let

$z_{i}=\beta^{i} \exp \left(A_{i} x+\left(C_{i}+\frac{B_{i}^{2}}{2\left(1-\rho^{2}\right)}\right) \eta+\left(\frac{1}{2}\left(\frac{\gamma_{i, 1}^{2}}{1-\rho_{\eta}^{2}}-\frac{2 \gamma_{i, 1} \gamma_{i, 2}}{1-\rho_{\eta} \rho^{2}}+\frac{\gamma_{i, 2}^{2}}{1-\rho^{4}}\right)+F_{i}\right) \omega^{2}\right)$

so that $\mathbf{E} y_{t}=\sum_{i=1}^{\infty} z_{i}$. Then

$$
\left|\frac{z_{i+1}}{z_{i}}\right|=\beta \exp \left(\begin{array}{c}
\widetilde{A} x+\left(\widetilde{C}_{i}+\frac{B_{i+1}^{2}-B_{i}^{2}}{2\left(1-\rho^{2}\right)}\right) \eta \\
+\left(\frac{1}{2}\left(\frac{\gamma_{i+1,1}^{2}-\gamma_{i, 1}^{2}}{1-\rho_{\eta}^{2}}-\frac{2\left(\gamma_{i+1,1} \gamma_{i+1,2}-\gamma_{i, 1} \gamma_{i, 2}\right)}{1-\rho_{\eta} \rho^{2}}+\frac{\gamma_{i+1,2}^{2}-\gamma_{i, 2}^{2}}{1-\rho^{4}}\right)+\widetilde{F}_{i}\right) \omega^{2}
\end{array}\right) .
$$

The parameters $\widetilde{A}, \widetilde{C}_{i}$, and $\widetilde{F}_{i}$ are the same as in Section A.3. Since Section A.3 also shows that $\lim _{i \rightarrow \infty} \widetilde{B}_{i}=\lim _{i \rightarrow \infty} \widetilde{D}_{i}=0$, it follows naturally (or after much tedious manipulation) that this result also implies that

$$
\begin{gathered}
\lim _{i \rightarrow \infty}\left(B_{i+1}^{2}-B_{i}^{2}\right)=\lim _{i \rightarrow \infty}\left(D_{i+1}-D_{i}\right)=0 \\
\lim _{i \rightarrow \infty}\left(\gamma_{i+1,1}^{2}-\gamma_{i, 1}^{2}\right)=\lim _{i \rightarrow \infty}\left(\gamma_{i+1,1} \gamma_{i+1,2}-\gamma_{i, 1} \gamma_{i, 2}\right)=\lim _{i \rightarrow \infty}\left(\gamma_{i+1,2}^{2}-\gamma_{i, 2}^{2}\right)=0
\end{gathered}
$$

This completes the proof.

\section{B.2.2 Risk-free rate}

The price of a risk-free bond, $p_{t}^{r f}$, is given by

$$
p_{t}^{r f}=\mathbf{E}_{t}\left(\beta\left(\frac{c_{t+1}}{c_{t}}\right)^{-\gamma}\right), \quad \text { or } \quad p_{t}^{r f}=\beta \mathbf{E}_{t} \exp \left(-\gamma x_{t+1}\right) .
$$

Substituting out $x_{t+1}$ gives

$$
p_{t}^{r f}=\beta \mathbf{E}_{t} \exp \left(-\gamma\left(x+\rho\left(x_{t}-x\right)+\sqrt{\left(\eta+\rho_{\eta}\left(\eta_{t}-\eta\right)+\omega \varepsilon_{\eta, t+1}\right)} \varepsilon_{t+1}\right)\right) .
$$

Expressing the expectation using integrals gives

$$
p_{t}^{r f}=\beta \exp \left(-\gamma x-\gamma \rho\left(x_{t}-x\right)\right) \int_{\varepsilon_{\eta, \mathbf{t}+\mathbf{1}}} \int_{\varepsilon_{\mathbf{t}+\mathbf{1}}} \exp \left(-\gamma \sqrt{\left(\eta+\rho_{\eta}\left(\eta_{t}-\eta\right)+\omega \varepsilon_{\eta, t+1}\right)} \varepsilon_{t+1}\right) d F d F_{\eta}
$$

Taking expectations with respect to $\varepsilon_{t+1}$ gives

$$
p_{t}^{r f}=\beta \exp \left(-\gamma x-\gamma \rho\left(x_{t}-x\right)\right) \int_{\varepsilon_{\eta, \mathbf{t}+\mathbf{1}}} \exp \left(\frac{\gamma^{2}}{2}\left(\eta+\rho_{\eta}\left(\eta_{t}-\eta\right)+\omega \varepsilon_{\eta, t+1}\right)\right) d F_{\eta}
$$


and bringing time $t$ terms outside the integral gives

$$
p_{t}^{r f}=\beta \exp \left(-\gamma x-\gamma \rho\left(x_{t}-x\right)+\frac{1}{2} \gamma^{2} \eta+\frac{\gamma^{2} \rho_{\eta}}{2}\left(\eta_{t}-\eta\right)\right) \int_{\varepsilon_{\eta, \mathbf{t}+\mathbf{1}}} \exp \left(\frac{\gamma^{2}}{2} \omega \varepsilon_{\eta, t+1}\right) d F_{\eta}
$$

Taking expectations with respect to $\varepsilon_{\eta, t+1}$ gives

$$
p_{t}^{r f}=\beta \exp \left(-\gamma x-\gamma \rho\left(x_{t}-x\right)+\frac{1}{2} \gamma^{2} \eta+\frac{\gamma^{2} \rho_{\eta}}{2}\left(\eta_{t}-\eta\right)+\frac{\gamma^{4}}{8} \omega^{2}\right) .
$$

Since the risk-free rate is the inverse of the price of the risk-free bond, $R_{t+1}^{r f}=\left(p_{t}^{r f}\right)^{-1}$, the risk-free rate is

$$
R_{t+1}^{r f}=\beta^{-1} \exp \left(\gamma x+\gamma \rho\left(x_{t}-x\right)-\frac{1}{2} \gamma^{2} \eta-\frac{\gamma^{2} \rho_{\eta}}{2}\left(\eta_{t}-\eta\right)-\frac{\gamma^{4}}{8} \omega^{2}\right) .
$$

\section{B.2.3 Conditional-expected return on equity}

The conditional-expected return on equity, $\mathbf{E}_{t} R_{t+1}$, is defined as

$$
\mathbf{E}_{t}\left(\frac{d_{t+1}+p_{t+1}}{p_{t}}\right)
$$

and can be rewritten as

$$
\frac{\mathbf{E}_{t} \exp \left(x_{t+1}\right)+\mathbf{E}_{t}\left(y_{t+1} \exp \left(x_{t+1}\right)\right)}{y_{t}}
$$

Thus, there are two expectations terms to evaluate. The first, $\mathbf{E}_{t} \exp \left(x_{t+1}\right)$, is

$$
\exp \left(x+\rho\left(x_{t}-x\right)+\frac{1}{2} \eta+\frac{\rho_{\eta}}{2}\left(\eta_{t}-\eta\right)+\frac{1}{8} \omega^{2}\right) .
$$

The second, $\mathbf{E}_{t}\left(y_{t+1} \exp \left(x_{t+1}\right)\right)$, can initially be rewritten as

$$
\sum_{i=1}^{\infty} \beta^{i} \exp \left(Z_{i}+x\right) \mathbf{E}_{t} \exp \left(\left(B_{i}+1\right)\left(x_{t+1}-x\right)+D_{i}\left(\eta_{t+1}-\eta\right)\right)
$$

where

$$
Z_{i} \equiv A_{i} x+C_{i} \eta+F_{i} \omega^{2}
$$

Focusing on only the expectations term, substituting in for the exogenous processes and using the integral notation gives

$$
\begin{aligned}
& \exp \left(\left(B_{i}+1\right) \rho\left(x_{t}-x\right)+D_{i} \rho_{\eta}\left(\eta_{t}-\eta\right)\right) \\
& \times \int_{\varepsilon_{\eta, \mathbf{t}+\mathbf{1}}} \int_{\boldsymbol{\varepsilon}_{\mathbf{t}+\mathbf{1}}} \exp \left(\left(B_{i}+1\right) \sqrt{\left(\eta+\rho_{\eta}\left(\eta_{t}-\eta\right)+\omega \varepsilon_{\eta, t+1}\right)} \varepsilon_{t+1}\right) d F \exp \left(D_{i} \omega \varepsilon_{\eta, t+1}\right) d F_{\eta} .
\end{aligned}
$$


Taking expectations with respect to $\varepsilon_{t+1}$ gives

$$
\begin{aligned}
& \exp \left(\left(B_{i}+1\right) \rho\left(x_{t}-x\right)+\frac{1}{2}\left(B_{i}+1\right)^{2}\left(\eta+\rho_{\eta}\left(\eta_{t}-\eta\right)\right)\right) \\
& \times \int_{\varepsilon_{\eta, \mathbf{t}+\mathbf{1}}} \exp \left(\left(\frac{1}{2}\left(B_{i}+1\right)^{2}+D_{i}\right) \omega \varepsilon_{\eta, t+1}\right) d F_{\eta} .
\end{aligned}
$$

Next, taking expectations with respect to $\varepsilon_{\eta, t+1}$ gives

$$
\exp \left(\left(B_{i}+1\right) \rho\left(x_{t}-x\right)+\frac{1}{2}\left(B_{i}+1\right)^{2}\left(\eta+\rho_{\eta}\left(\eta_{t}-\eta\right)\right)+\frac{1}{2}\left(\frac{1}{2}\left(B_{i}+1\right)^{2}+D_{i}\right)^{2} \omega^{2}\right)
$$

The term, $\mathbf{E}_{t}\left(y_{t+1} \exp \left(x_{t+1}\right)\right)$, can therefore be written as:

$$
\sum_{i=1}^{\infty} \beta^{i} \exp \left(\begin{array}{c}
\left(A_{i}+1\right) x+\left(B_{i}+1\right) \rho\left(x_{t}-x\right) \\
+\left(C_{i}+\frac{1}{2}\left(B_{i}+1\right)^{2}\right) \eta+\frac{1}{2}\left(B_{i}+1\right)^{2} \rho_{\eta}\left(\eta_{t}-\eta\right) \\
+\left(F_{i}+\frac{1}{2}\left(\frac{1}{2}\left(B_{i}+1\right)^{2}+D_{i}\right)^{2}\right) \omega^{2}
\end{array}\right)
$$

\section{B.3 Low-order polynomial approximations}

This section derives approximate solutions for the price-dividend ratio using, first, the perturbation method (up to sixth order), and second, the Campbell and Shiller (1988) first-order approximation exploiting the normality of the shock processes.

\section{B.3.1 The perturbation solution}

The sixth order perturbation approximate solution is

$$
\begin{gathered}
y_{t}=g+g_{x} \widehat{x}_{t}+\frac{1}{2 !}\left(g_{\sigma^{2}}+g_{x^{2}} \widehat{x}_{t}^{2}\right)+\frac{1}{3 !}\left(3 g_{\sigma^{2} x} \widehat{x}_{t}+3 g_{\sigma^{2} \eta} \widehat{\eta}_{t}+g_{x^{3}} \widehat{x}_{t}^{3}\right) \\
+\frac{1}{4 !}\left(g_{\sigma^{4}}+6 g_{\sigma^{2} x^{2}} \widehat{x}_{t}^{2}+12 g_{\sigma^{2} x \eta} \widehat{x}_{t} \widehat{\eta}_{t}+g_{x^{4}} \widehat{x}_{t}^{4}\right) \\
+\frac{1}{5 !}\left(5 g_{\sigma^{4} x} \widehat{x}_{t}+5 g_{\sigma^{4} \eta} \widehat{\eta}_{t}+10 g_{\sigma^{2} x^{3}} \widehat{x}_{t}^{3}+30 g_{\sigma^{2} x^{2} \eta} \widehat{x}_{t}^{2} \widehat{\eta}_{t}+g_{x^{5}} \widehat{x}_{t}^{5}\right) \\
+\frac{1}{6 !}\left(g_{\sigma^{6}}+15 g_{\sigma^{4} x^{2}} \widehat{x}_{t}^{2}+30 g_{\sigma^{4} x \eta} \widehat{x}_{t} \widehat{\eta}_{t}+15 g_{\sigma^{4} \eta^{2}} \widehat{\eta}_{t}^{2}+60 g_{\sigma^{2} x^{3} \eta} \widehat{x}_{t}^{3} \widehat{\eta}_{t}+g_{x^{6}} \widehat{x}_{t}^{6}\right),
\end{gathered}
$$

where $\widehat{x}_{t} \equiv x_{t}-x, \widehat{\eta}_{t} \equiv \eta_{t}-\eta$, and

$$
\begin{array}{cc}
g_{\sigma^{4}}=\sum_{i=1}^{\infty} \beta^{i} \exp \left(A_{i} x\right) 12 \eta^{2} C_{i}^{2}, & g_{\sigma^{2} x^{2}}=\sum_{i=1}^{\infty} \beta^{i} \exp \left(A_{i} x\right) 2 \eta B_{i}^{2} C_{i}, \\
g_{\sigma^{2} x \eta}=\sum_{i=1}^{\infty} \beta^{i} \exp \left(A_{i} x\right) 2 B_{i} D_{i}, & g_{x^{4}}=\sum_{i=1}^{\infty} \beta^{i} \exp \left(A_{i} x\right) B_{i}^{4}, \\
g_{\sigma^{4} x}=\sum_{i=1}^{\infty} \beta^{i} \exp \left(A_{i} x\right) 12 \eta^{2} B_{i} C_{i}^{2}, & g_{\sigma^{4} \eta}=\sum_{i=1}^{\infty} \beta^{i} \exp \left(A_{i} x\right) 24 \eta C_{i} D_{i}, \\
g_{\sigma^{2} x^{3}}=\sum_{i=1}^{\infty} \beta^{i} \exp \left(A_{i} x\right) 2 \eta B_{i}^{3} C_{i}, & g_{\sigma^{2} x^{2} \eta}=\sum_{i=1}^{\infty} \beta^{i} \exp \left(A_{i} x\right) 2 B_{i}^{2} D_{i}, \\
g_{x^{5}}=\sum_{i=1}^{\infty} \beta^{i} \exp \left(A_{i} x\right) B_{i}^{5}, & g_{\sigma^{6}}=\sum_{i=1}^{\infty} \beta^{i} \exp \left(A_{i} x\right)\left(\eta^{3} C_{i}^{3}+720 F_{i} \omega^{2}\right), \\
g_{\sigma^{4} x^{2}}=\sum_{i=1}^{\infty} \beta^{i} \exp \left(A_{i} x\right) 12 \eta^{2} B_{i}^{2} C_{i}^{2}, & g_{\sigma^{4} x \eta}=\sum_{i=1}^{\infty} \beta^{i} \exp \left(A_{i} x\right) 24 \eta B_{i} C_{i} D_{i}, \\
g_{\sigma^{4} \eta^{2}}=\sum_{i=1}^{\infty} \beta^{i} \exp \left(A_{i} x\right) 24 D_{i}^{2}, & g_{\sigma^{2} x^{4}}=\sum_{i=1}^{\infty} \beta^{i} \exp \left(A_{i} x\right) 2 \eta B_{i}^{4} C_{i}, \\
g_{\sigma^{2} x^{3} \eta}=\sum_{i=1}^{\infty} \beta^{i} \exp \left(A_{i} x\right) 2 B_{i}^{3} D_{i}, & g_{x^{6}}=\sum_{i=1}^{\infty} \beta^{i} \exp \left(A_{i} x\right) B_{i}^{6} .
\end{array}
$$




\section{B.3.2 The first-order solution exploiting normality}

Defining the log-price-dividend ratio as $\widetilde{y}_{t} \equiv \log y_{t}$, we are searching for a solution of the form

$$
\widetilde{y}_{t}=\widetilde{y}+\kappa_{1}\left(x_{t}-x\right)+\kappa_{2}\left(\eta_{t}-\eta\right)
$$

where $\kappa_{1}$ and $\kappa_{2}$ are as yet undetermined coefficients. The Euler equation from the main text, in terms of the log-price-dividend ratio, is given by

$$
\exp \widetilde{y}_{t}=\beta \mathbf{E}_{t} \exp \left((1-\gamma) x_{t+1}+\log \left(1+\exp \widetilde{y}_{t+1}\right)\right)
$$

The first step is to take a first-order approximation of $\log \left(1+\exp \widetilde{y}_{t+1}\right)$ around $\widetilde{y}_{t+1}=\widetilde{y}$ as follows

$$
\log \left(1+\exp \widetilde{y}_{t+1}\right) \simeq \log (1+y)+\frac{y}{1+y}\left(\widetilde{y}_{t+1}-\widetilde{y}\right)
$$

and substitute this approximation into the Euler equation:

$$
\exp \widetilde{y}_{t}=\beta \mathbf{E}_{t} \exp \left((1-\gamma) x_{t+1}+\log (1+y)+\frac{y}{1+y}\left(\widetilde{y}_{t+1}-\widetilde{y}\right)\right) \text {. }
$$

Moving terms outside the expectations operator gives

$$
\exp \widetilde{y}_{t}=\beta(1+y) \mathbf{E}_{t} \exp \left((1-\gamma) x_{t+1}+\frac{y}{1+y}\left(\widetilde{y}_{t+1}-\widetilde{y}\right)\right)
$$

and, substituting in equation (B.4) with undetermined coefficients $\kappa_{1}$ and $\kappa_{2}$, changes the right-hand side to

$$
\beta(1+y) \mathbf{E}_{t} \exp \left((1-\gamma) x_{t+1}+\frac{y}{1+y}\left(\kappa_{1}\left(x_{t+1}-x\right)+\kappa_{2}\left(\eta_{t+1}-\eta\right)\right)\right) .
$$

Collecting the $\left(x_{t+1}-x\right)$ terms gives

$$
\beta \exp ((1-\gamma) x)(1+y) \mathbf{E}_{t} \exp \left(\left(1-\gamma+\frac{y \kappa_{1}}{1+y}\right)\left(x_{t+1}-x\right)+\frac{y \kappa_{2}}{1+y}\left(\eta_{t+1}-\eta\right)\right),
$$

and substituting in the process for dividends gives

$$
\beta \exp ((1-\gamma) x)(1+y) \mathbf{E}_{t} \exp \left(\left(1-\gamma+\frac{y \kappa_{1}}{1+y}\right)\left(\rho\left(x_{t}-x\right)+\sqrt{\eta_{t+1}} \varepsilon_{t+1}\right)+\frac{y \kappa_{2}}{1+y}\left(\eta_{t+1}-\eta\right)\right)
$$

which can then be rewritten as

$$
\begin{aligned}
& \beta \exp \left((1-\gamma) x+\left(1-\gamma+\frac{y \kappa_{1}}{1+y}\right) \rho\left(x_{t}-x\right)\right)(1+y) \\
& \times \mathbf{E}_{t} \exp \left(\left(1-\gamma+\frac{y \kappa_{1}}{1+y}\right) \sqrt{\eta_{t+1}} \varepsilon_{t+1}+\frac{y \kappa_{2}}{1+y}\left(\eta_{t+1}-\eta\right)\right) .
\end{aligned}
$$


Taking only expectations over $\varepsilon_{t+1}$ gives

$$
\begin{aligned}
& \beta \exp \left((1-\gamma) x+\left(1-\gamma+\frac{y \kappa_{1}}{1+y}\right) \rho\left(x_{t}-x\right)\right)(1+y) \\
& \times \mathbf{E}_{t} \exp \left(\frac{1}{2}\left(1-\gamma+\frac{y \kappa_{1}}{1+y}\right)^{2} \eta_{t+1}+\frac{y \kappa_{2}}{1+y}\left(\eta_{t+1}-\eta\right)\right),
\end{aligned}
$$

Collecting $\left(\eta_{t+1}-\eta\right)$ terms gives

$$
\begin{aligned}
& \beta \exp \left((1-\gamma) x+\left(1-\gamma+\frac{y \kappa_{1}}{1+y}\right) \rho\left(x_{t}-x\right)+\frac{1}{2}\left(1-\gamma+\frac{y \kappa_{1}}{1+y}\right)^{2} \eta\right)(1+y) \\
& \times \mathbf{E}_{t} \exp \left(\left(\frac{1}{2}\left(1-\gamma+\frac{y \kappa_{1}}{1+y}\right)^{2}+\frac{y \kappa_{2}}{1+y}\right)\left(\eta_{t+1}-\eta\right)\right)
\end{aligned}
$$

and substituting in the stochastic volatility process gives

$$
\begin{aligned}
& \beta \exp \left(\begin{array}{c}
(1-\gamma) x+\left(1-\gamma+\frac{y \kappa_{1}}{1+y}\right) \rho\left(x_{t}-x\right)+\frac{1}{2}\left(1-\gamma+\frac{y \kappa_{1}}{1+y}\right)^{2} \eta \\
+\left(\frac{1}{2}\left(1-\gamma+\frac{y \kappa_{1}}{1+y}\right)^{2}+\frac{y \kappa_{2}}{1+y}\right) \rho_{\eta}\left(\eta_{t}-\eta\right)
\end{array}\right)(1+y) \\
& \times \mathbf{E}_{t} \exp \left(\left(\frac{1}{2}\left(1-\gamma+\frac{y \kappa_{1}}{1+y}\right)^{2}+\frac{y \kappa_{2}}{1+y}\right) \omega \varepsilon_{\eta, t+1}\right) .
\end{aligned}
$$

Taking expectations gives

$$
\exp \left(\begin{array}{c}
\log \beta+(1-\gamma) x+\log (1+y)+\frac{1}{2}\left(1-\gamma+\frac{y \kappa_{1}}{1+y}\right)^{2} \eta \\
+\frac{1}{2}\left(\frac{1}{2}\left(1-\gamma+\frac{y \kappa_{1}}{1+y}\right)^{2}+\frac{y \kappa_{2}}{1+y}\right)^{2} \omega^{2} \\
+\left(1-\gamma+\frac{y \kappa_{1}}{1+y}\right) \rho\left(x_{t}-x\right) \\
+\left(\frac{1}{2}\left(1-\gamma+\frac{y \kappa_{1}}{1+y}\right)^{2}+\frac{y \kappa_{2}}{1+y}\right) \rho_{\eta}\left(\eta_{t}-\eta\right)
\end{array}\right)
$$

The left-hand side of equation (B.5) is

$$
\exp \left(\widetilde{y}+\kappa_{1}\left(x_{t}-x\right)+\kappa_{2}\left(\eta_{t}-\eta\right)\right)
$$

Hence, it is possible to match coefficients. First,

$$
\kappa_{1}=\left(1-\gamma+\frac{y \kappa_{1}}{1+y}\right) \rho, \quad \text { which implies } \quad \kappa_{1}=\frac{(1-\gamma) \rho}{1-\frac{y \rho}{1+y}} .
$$

Second,

$$
\kappa_{2}=\left(\frac{1}{2}\left(1-\gamma+\frac{y \kappa_{1}}{1+y}\right)^{2}+\frac{y \kappa_{2}}{1+y}\right) \rho_{\eta}
$$


which implies

$$
\kappa_{2}=\frac{\left(1-\gamma+\frac{y \kappa_{1}}{1+y}\right)^{2} \rho_{\eta}}{2\left(1-\frac{y \rho_{\eta}}{1+y}\right)}
$$

Third,

$$
\begin{aligned}
\log y= & \log \beta+(1-\gamma) x+\log (1+y)+\frac{1}{2}\left((1-\gamma)+\frac{y}{1+y} \kappa_{1}\right)^{2} \eta \\
& +\frac{1}{2}\left(\frac{1}{2}\left((1-\gamma)+\frac{y}{1+y} \kappa_{1}\right)^{2}+\frac{y}{1+y} \kappa_{2}\right)^{2} \omega^{2} .
\end{aligned}
$$

Substituting for $\kappa_{1}$ and $\kappa_{2}, y$ solves

$$
\frac{y}{1+y}=\beta \exp \left((1-\gamma) x+\frac{(1-\gamma)^{2}(1+y)^{2} \eta}{2(1+(1-\rho) y)^{2}}+\frac{(1-\gamma)^{4}(1+y)^{6} \omega^{2}}{8(1+(1-\rho) y)^{4}\left(1+\left(1-\rho_{\eta}\right) y\right)^{2}}\right)
$$

\section{B.4 Bansal \& Yaron without recursive preferences}

The model in the main text is an extension of Burnside (1998) to allow for stochastic volatility in the dividend growth process. Alternatively, it may be of interest to solve a variant of the model presented in Bansal and Yaron (2004). Relative to the model in the main text, the model in Bansal and Yaron (2004) features long-run risk, recursive preferences and differential processes for consumption and dividends. While it is not possible to find a closed-form solution with recursive preferences, it is possible to find the closed-form solution of the model with CRRA preferences, plus long-run risk and separate consumption and dividend processes. In this section, I solve the model, and use the calibration in Bansal and Yaron (2004) to report some several moments of the model solution.

\section{B.4.1 The model}

The Euler equation is as it was in equation (5):

$$
c_{t}^{-\gamma} p_{t}=\mathbf{E}_{t} \beta c_{t+1}^{-\gamma}\left(p_{t+1}+d_{t+1}\right)
$$

Denoting the log-growth rate of consumption and dividends as

$$
g_{c, t} \equiv \log \left(c_{t} / c_{t-1}\right) \quad \text { and } \quad g_{d, t} \equiv \log \left(d_{t} / d_{t-1}\right)
$$

respectively, the forward iterated Euler equation can be rewritten as follows:

$$
y_{t}=\sum_{i=1}^{\infty} \beta^{i} \mathbf{E}_{t} \exp \left(\sum_{j=1}^{i}\left(g_{d, t+j}-\gamma g_{c, t+j}\right)\right)
$$


Notice that if $g_{c, t}=g_{d, t}$ as in the main text, we recover (7). The exogenous processes for consumption and dividends are modelled as follows

$$
\begin{gathered}
g_{c, t+1}=\mu_{c}+x_{t}+\sigma_{t} \varepsilon_{c, t+1}, \\
g_{d, t+1}=\mu_{d}+\phi x_{t}+\varphi_{d} \sigma_{t} \varepsilon_{d, t+1},
\end{gathered}
$$

where

$$
\begin{gathered}
x_{t+1}=\rho x_{t}+\varphi_{x} \sigma_{t} \varepsilon_{x, t+1} \\
\sigma_{t+1}^{2}=\sigma^{2}+\rho_{\sigma}\left(\sigma_{t}^{2}-\sigma^{2}\right)+\sigma_{\omega} \varepsilon_{\sigma, t+1} .
\end{gathered}
$$

This follows the specification in Bansal and Yaron (2004). The four shocks, $\varepsilon_{c, t+1}, \varepsilon_{d, t+1}, \varepsilon_{x, t+1}$, and $\varepsilon_{\sigma, t+1}$ are all independent standard normals. Notice that the notation is slightly altered from the main text $-x_{t}$ is no longer the growth rate of dividends but the persistentpredictable component of both consumption and dividend growth. The timing of stochastic volatility is also slightly altered from the main text. The two state variables are $x_{t}$ and $\sigma_{t}^{2}$.

\section{B.4.2 The solution}

The solution is of the form

$$
y_{t}=\sum_{i=1}^{\infty} \beta^{i} \exp \left(A_{i}^{B Y}+B_{i}^{B Y} x_{t}+C_{i}^{B Y} \sigma^{2}+D_{i}^{B Y}\left(\sigma_{t}^{2}-\sigma^{2}\right)+F_{i}^{B Y} \sigma_{\omega}^{2}\right)
$$

where $A_{i}^{B Y}, B_{i}^{B Y}, C_{i}^{B Y}, D_{i}^{B Y}$, and $F_{i}^{B Y}$ are coefficients to be determined. Substituting the consumption and dividend process into the expectations component of equation (B.6) and collecting like terms gives

$$
\mathbf{E}_{t} \exp \left(i\left(\mu_{d}-\gamma \mu_{c}\right)+(\phi-\gamma) \sum_{j=1}^{i} x_{t+j-1}+\sum_{j=1}^{i} \sigma_{t+j-1}\left(\varphi_{d} \varepsilon_{d, t+j}-\gamma \varepsilon_{c, t+j}\right)\right)
$$

where it becomes immediately clear that

$$
A_{i}^{B Y} \equiv i\left(\mu_{d}-\gamma \mu_{c}\right)
$$

As I progress with the solution, I will focus only on the expectations term that is yet to be determined. Thus, I drop $A_{i}^{B Y}$ and focus on

$$
\mathbf{E}_{t} \exp \left((\phi-\gamma) x_{t}+(\phi-\gamma) \sum_{j=1}^{i-1} x_{t+j}+\sum_{j=1}^{i} \sigma_{t+j-1}\left(\varphi_{d} \varepsilon_{d, t+j}-\gamma \varepsilon_{c, t+j}\right)\right)
$$

Iterating forward the process for $x_{t}$ gives

$$
x_{t+j}=\rho^{j} x_{t}+\varphi_{x} \sum_{k=1}^{j} \rho^{j-k} \sigma_{t+k-1} \varepsilon_{x, t+k}
$$


Substituting this into the above expression and collecting terms gives

$$
\mathbf{E}_{t} \exp \left(\begin{array}{c}
(\phi-\gamma) \sum_{j=1}^{i} \rho^{j-1} x_{t}+\varphi_{x}(\phi-\gamma) \sum_{j=1}^{i-1}\left(\sum_{k=1}^{j} \rho^{j-k} \sigma_{t+k-1} \varepsilon_{x, t+k}\right) \\
+\sum_{j=1}^{i} \sigma_{t+j-1}\left(\varphi_{d} \varepsilon_{d, t+j}-\gamma \varepsilon_{c, t+j}\right)
\end{array}\right)
$$

which makes it clear that

$$
B_{i}^{B Y} \equiv(\phi-\gamma) \frac{1-\rho^{i}}{1-\rho}
$$

leaving only the following:

$$
\mathbf{E}_{t} \exp \left(\varphi_{x}(\phi-\gamma) \sum_{j=1}^{i-1}\left(\sum_{k=1}^{j} \rho^{j-k} \sigma_{t+k-1} \varepsilon_{x, t+k}\right)+\sum_{j=1}^{i} \sigma_{t+j-1}\left(\varphi_{d} \varepsilon_{d, t+j}-\gamma \varepsilon_{c, t+j}\right)\right)
$$

Next, it is necessary to collect $\varepsilon_{x}$ terms with the same time subscript, which requires rewriting the expression above as follows:

$$
\mathbf{E}_{t} \exp \left(\frac{(\phi-\gamma) \varphi_{x}}{1-\rho} \sum_{j=1}^{i-1}\left(1-\rho^{i-j}\right) \sigma_{t+j-1} \varepsilon_{x, t+j}+\sum_{j=1}^{i} \sigma_{t+j-1}\left(\varphi_{d} \varepsilon_{d, t+j}-\gamma \varepsilon_{c, t+j}\right)\right)
$$

Taking expectations over the three fundamental (and independent) shocks, $\varepsilon_{c}, \varepsilon_{d}$, and $\varepsilon_{x}$, (but not $\varepsilon_{\sigma}$ yet) gives

$$
\mathbf{E}_{t} \exp \left(\frac{1}{2}\left(\frac{(\phi-\gamma) \varphi_{x}}{1-\rho}\right)^{2} \sum_{j=1}^{i-1}\left(1-\rho^{i-j}\right)^{2} \sigma_{t+j-1}^{2}+\frac{1}{2}\left(\varphi_{d}^{2}+\gamma^{2}\right) \sum_{j=1}^{i} \sigma_{t+j-1}^{2}\right)
$$

Removing the $\sigma_{t}^{2}$ term from the expectations operator gives

$$
\begin{aligned}
& \exp \left(\frac{1}{2}\left(\left(\frac{(\phi-\gamma) \varphi_{x}}{1-\rho}\right)^{2}\left(1-\rho^{i-1}\right)^{2}+\left(\varphi_{d}^{2}+\gamma^{2}\right)\right) \sigma_{t}^{2}\right) \\
& \times \mathbf{E}_{t} \exp \left(\frac{1}{2}\left(\frac{(\phi-\gamma) \varphi_{x}}{1-\rho}\right)^{2} \sum_{j=2}^{i-1}\left(1-\rho^{i-j}\right)^{2} \sigma_{t+j-1}^{2}+\frac{1}{2}\left(\varphi_{d}^{2}+\gamma^{2}\right) \sum_{j=2}^{i} \sigma_{t+j-1}^{2}\right) .
\end{aligned}
$$

Iterating forward the stochastic volatility process gives

$$
\sigma_{t+j-1}^{2}=\sigma^{2}+\rho_{\sigma}^{j-1}\left(\sigma_{t}^{2}-\sigma^{2}\right)+\sigma_{\omega} \sum_{k=1}^{j-1} \rho_{\sigma}^{j-1-k} \varepsilon_{\sigma, t+k}
$$

and substituting into the above expression and collecting terms for $\sigma^{2}$ and $\left(\sigma_{t}^{2}-\sigma^{2}\right)$ gives

$$
\mathbf{E}_{t} \exp \left(\begin{array}{c}
\frac{1}{2}\left(\left(\frac{(\phi-\gamma) \varphi_{x}}{1-\rho}\right)^{2} \sum_{j=1}^{i-1}\left(1-\rho^{i-j}\right)^{2}+\sum_{j=1}^{i}\left(\varphi_{d}^{2}+\gamma^{2}\right)\right) \sigma^{2} \\
+\frac{1}{2}\left(\left(\frac{(\phi-\gamma) \varphi_{x}}{1-\rho}\right)^{2} \sum_{j=1}^{i-1}\left(1-\rho^{i-j}\right)^{2} \rho_{\sigma}^{j-1}+\left(\varphi_{d}^{2}+\gamma^{2}\right) \sum_{j=1}^{i} \rho_{\sigma}^{j-1}\right)\left(\sigma_{t}^{2}-\sigma^{2}\right) \\
+\frac{1}{2}\left(\frac{(\phi-\gamma) \varphi_{x}}{1-\rho}\right)^{2} \sum_{j=2}^{i-1}\left(1-\rho^{i-j}\right)^{2} \sigma_{\omega} \sum_{k=1}^{j-1} \rho_{\sigma}^{j-1-k} \varepsilon_{\sigma, t+k} \\
+\frac{1}{2}\left(\varphi_{d}^{2}+\gamma^{2}\right) \sum_{j=2}^{i} \sigma_{\omega} \sum_{k=1}^{j-1} \rho_{\sigma}^{j-1-k} \varepsilon_{\sigma, t+k}
\end{array}\right) .
$$


The first line above can be rewritten as $C_{i}^{B Y} \sigma^{2}$, where

$$
C_{i}^{B Y} \equiv \frac{1}{2}\left(\left(\frac{(\phi-\gamma) \varphi_{x}}{1-\rho}\right)^{2}\left((i-1)-2 \rho \frac{1-\rho^{i-1}}{1-\rho}+\rho^{2} \frac{1-\rho^{2(i-1)}}{1-\rho^{2}}\right)+i\left(\varphi_{d}^{2}+\gamma^{2}\right)\right)
$$

and the second line can be rewritten as $D_{i}^{B Y}\left(\sigma_{t}^{2}-\sigma^{2}\right)$, where

$$
D_{i}^{B Y} \equiv \frac{1}{2}\left(\begin{array}{c}
\left(\frac{(\phi-\gamma) \varphi_{x}}{1-\rho}\right)^{2}\left(\frac{1-\rho_{\sigma}^{i-1}}{1-\rho_{\sigma}}-2 \rho^{i-1} \frac{1-\left(\rho^{-1} \rho_{\sigma}\right)^{i-1}}{1-\left(\rho^{-1} \rho_{\sigma}\right)}+\rho^{2(i-1)} \frac{1-\left(\rho^{-2} \rho_{\sigma}\right)^{i-1}}{1-\left(\rho^{-2} \rho_{\sigma}\right)}\right) \\
+\frac{1-\rho_{\sigma}^{i}}{1-\rho_{\sigma}}\left(\varphi_{d}^{2}+\gamma^{2}\right)
\end{array}\right)
$$

This leaves the expression

$$
\mathbf{E}_{t} \exp \left(\begin{array}{c}
\frac{\sigma_{\omega}}{2}\left(\frac{(\phi-\gamma) \varphi_{x}}{1-\rho}\right)^{2} \sum_{j=1}^{i-2}\left(1-\rho^{i-j}\right)^{2}\left(\sum_{k=1}^{j} \rho_{\sigma}^{j-k} \varepsilon_{\sigma, t+k}\right) \\
+\frac{\sigma_{\omega}}{2}\left(\varphi_{d}^{2}+\gamma^{2}\right) \sum_{j=1}^{i-1}\left(\sum_{k=1}^{j} \rho_{\sigma}^{j-k} \varepsilon_{\sigma, t+k}\right)
\end{array}\right)
$$

yet to be evaluated, where, for convenience, I have rewritten the indexing in the summation. Next, it is necessary to collect $\varepsilon_{\sigma}$ terms with the same time subscript, rewriting the expression as follows:

$$
\mathbf{E}_{t} \exp \left(\begin{array}{c}
\frac{\sigma_{\omega}}{2} \varkappa_{1} \sum_{j=1}^{i-2}\left(\sum_{k=1}^{i-j-1}\left(1-\rho^{i-j-k+1}\right)^{2} \rho_{\sigma}^{k-1}\right) \varepsilon_{\sigma, t+j} \\
+\frac{\sigma_{\omega}}{2}\left(\varphi_{d}^{2}+\gamma^{2}\right) \sum_{j=1}^{i-1}\left(\sum_{k=1}^{i-j} \rho_{\sigma}^{k-1}\right) \varepsilon_{\sigma, t+j}
\end{array}\right)
$$

And, using the standard results of geometric progressions gives

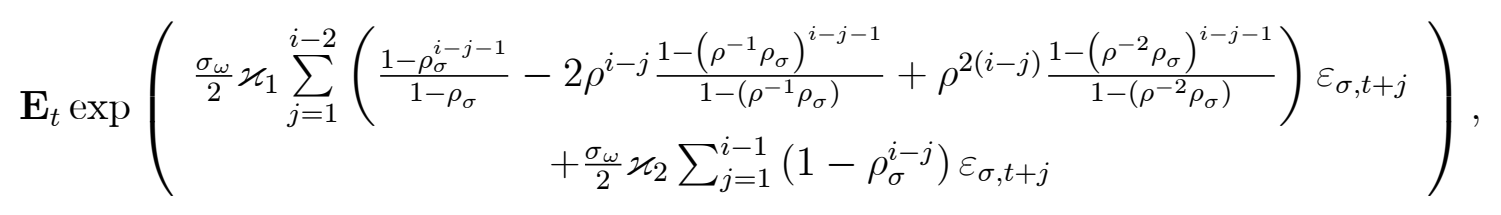

where

$$
\varkappa_{1} \equiv\left(\frac{(\phi-\gamma) \varphi_{x}}{1-\rho}\right)^{2} \quad \text { and } \quad \varkappa_{2} \equiv\left(\frac{\varphi_{d}^{2}+\gamma^{2}}{1-\rho_{\sigma}}\right) .
$$

Combining $\varepsilon_{\sigma}$ terms with the same time subscripts gives

$$
\mathbf{E}_{t} \exp \left(\begin{array}{c}
\frac{\sigma_{\omega}}{2} \sum_{j=1}^{i-2}\left(\varkappa_{1}\left(\frac{1-\rho_{\sigma}^{i-j-1}}{1-\rho_{\sigma}}-2 \rho^{i-j} \frac{1-\left(\rho^{-1} \rho_{\sigma}\right)^{i-j-1}}{1-\left(\rho^{-1} \rho_{\sigma}\right)}+\rho^{2(i-j)} \frac{1-\left(\rho^{-2} \rho_{\sigma}\right)^{i-j-1}}{1-\left(\rho^{-2} \rho_{\sigma}\right)}\right)+\varkappa_{2}\left(1-\rho_{\sigma}^{i-j}\right)\right) \varepsilon_{\sigma, t+j} \\
+\frac{1}{2} \sigma_{\omega} \varkappa_{2}\left(1-\rho_{\sigma}\right) \varepsilon_{\sigma, t+i-1}
\end{array}\right)
$$

Using standard results for random variables gives

$$
\mathbf{E}_{t} \exp \left(\begin{array}{c}
\frac{\sigma_{\omega}^{2}}{8} \sum_{j=1}^{i-2}\left(\varkappa_{1}\left(\frac{1-\rho_{\sigma}^{i-j-1}}{1-\rho_{\sigma}}-2 \rho^{i-j} \frac{1-\left(\rho^{-1} \rho_{\sigma}\right)^{i-j-1}}{1-\left(\rho^{-1} \rho_{\sigma}\right)}+\rho^{2(i-j)} \frac{1-\left(\rho^{-2} \rho_{\sigma}\right)^{i-j-1}}{1-\left(\rho^{-2} \rho_{\sigma}\right)}\right)+\varkappa_{2}\left(1-\rho_{\sigma}^{i-j}\right)\right)^{2} \\
+\frac{1}{8} \sigma_{\omega}^{2} \varkappa_{2}^{2}\left(1-\rho_{\sigma}\right)^{2}
\end{array}\right) .
$$


The final task is to rewriting the expression

$\sum_{j=1}^{i-2}\left(\varkappa_{1}\left(\frac{1-\rho_{\sigma}^{i-j-1}}{1-\rho_{\sigma}}-2 \rho^{i-j} \frac{1-\left(\rho^{-1} \rho_{\sigma}\right)^{i-j-1}}{1-\left(\rho^{-1} \rho_{\sigma}\right)}+\rho^{2(i-j)} \frac{1-\left(\rho^{-2} \rho_{\sigma}\right)^{i-j-1}}{1-\left(\rho^{-2} \rho_{\sigma}\right)}\right)+\varkappa_{2}\left(1-\rho_{\sigma}^{i-j}\right)\right)^{2}$,

more parsimoniously. To do this, it is first convenient to reverse the indexation for $j=1, \ldots, i$ by rewriting $i-j-1=j$ as follows:

$$
\sum_{j=1}^{i-2}\left(\varkappa_{1}\left(\frac{1-\rho_{\sigma}^{j}}{1-\rho_{\sigma}}-2 \rho^{j+1} \frac{1-\left(\rho^{-1} \rho_{\sigma}\right)^{j}}{1-\left(\rho^{-1} \rho_{\sigma}\right)}+\rho^{2(j+1)} \frac{1-\left(\rho^{-2} \rho_{\sigma}\right)^{j}}{1-\left(\rho^{-2} \rho_{\sigma}\right)}\right)+\varkappa_{2}\left(1-\rho_{\sigma}^{j+1}\right)\right)^{2},
$$

Next, we can rewrite the expression as follows

$$
\sum_{j=1}^{i-2}\left(\varkappa_{1}\left(\chi_{1}+\chi_{2} \rho_{\sigma}^{j-1}+\chi_{3} \rho^{j-1}+\chi_{4} \rho^{2(j-1)}\right)+\varkappa_{2}\left(1+\chi_{5} \rho_{\sigma}^{j-1}\right)\right)^{2}
$$

where

$$
\begin{gathered}
\chi_{1} \equiv \frac{1}{1-\rho_{\sigma}}, \chi_{2} \equiv-\rho_{\sigma}\left(\frac{1}{1-\rho_{\sigma}}-\frac{2 \rho^{2}}{\rho-\rho_{\sigma}}+\frac{\rho^{4}}{\rho^{2}-\rho_{\sigma}}\right) \\
\chi_{3} \equiv-\frac{2 \rho^{3}}{\rho-\rho_{\sigma}}, \chi_{4} \equiv \frac{\rho^{6}}{\rho^{2}-\rho_{\sigma}}, \chi_{5} \equiv-\rho_{\sigma}^{2} .
\end{gathered}
$$

Multiplying out the squared term once gives

$$
\sum_{j=1}^{i-2}\left(\begin{array}{c}
\varkappa_{1}^{2}\left(\chi_{1}+\chi_{2} \rho_{\sigma}^{j-1}+\chi_{3} \rho^{j-1}+\chi_{4} \rho^{2(j-1)}\right)^{2}+\varkappa_{2}^{2}\left(1+\chi_{5} \rho_{\sigma}^{j-1}\right)^{2} \\
+2 \varkappa_{1} \varkappa_{2}\left(\chi_{1}+\chi_{2} \rho_{\sigma}^{j-1}+\chi_{3} \rho^{j-1}+\chi_{4} \rho^{2(j-1)}\right)\left(1+\chi_{5} \rho_{\sigma}^{j-1}\right)
\end{array}\right)
$$

and a second time gives

$$
\sum_{j=1}^{i-2}\left(\begin{array}{c}
\varkappa_{1}^{2}\left(\begin{array}{c}
\chi_{1}^{2}+\chi_{2}^{2} \rho_{\sigma}^{2(j-1)}+\left(\chi_{3}^{2}+2 \chi_{1} \chi_{4}\right) \rho^{2(j-1)}+\chi_{4}^{2} \rho^{4(j-1)}+2 \chi_{1} \chi_{2} \rho_{\sigma}^{j-1} \\
+2 \chi_{1} \chi_{3} \rho^{j-1}+2 \chi_{2} \chi_{3}\left(\rho_{\sigma} \rho\right)^{j-1}+2 \chi_{2} \chi_{4}\left(\rho_{\sigma} \rho^{2}\right)^{j-1}+2 \chi_{3} \chi_{4} \rho^{3(j-1)}
\end{array}\right) \\
+\varkappa_{2}^{2}\left(1+2 \chi_{5} \rho_{\sigma}^{j-1}+\chi_{5}^{2} \rho_{\sigma}^{2(j-1)}\right) \\
+2 \varkappa_{1} \varkappa_{2}\left(\begin{array}{c}
\chi_{1}+\left(\chi_{2}+\chi_{1} \chi_{5}\right) \rho_{\sigma}^{j-1}+\chi_{3} \rho^{j-1}+\chi_{4} \rho^{2(j-1)} \\
+\chi_{2} \chi_{5} \rho_{\sigma}^{2(j-1)}+\chi_{3} \chi_{5}\left(\rho_{\sigma} \rho\right)^{j-1}+\chi_{4} \chi_{5}\left(\rho_{\sigma} \rho^{2}\right)^{j-1}
\end{array}\right)
\end{array}\right),
$$

Using the standard results of geometric progressions for the final time gives

$$
\begin{gathered}
\varkappa_{1}^{2}\left(\begin{array}{c}
(i-2) \chi_{1}^{2}+\chi_{2}^{2} \frac{1-\rho_{\sigma}^{2(i-2)}}{1-\rho_{\sigma}^{2}}+\left(\chi_{3}^{2}+2 \chi_{1} \chi_{4}\right) \frac{1-\rho^{2(i-2)}}{1-\rho^{2}}+\chi_{4}^{2} \frac{1-\rho^{4(i-2)}}{1-\rho^{4}}+2 \chi_{1} \chi_{2} \frac{1-\rho_{\sigma}^{i-2}}{1-\rho_{\sigma}} \\
+2 \chi_{1} \chi_{3} \frac{1-\rho^{i-2}}{1-\rho}+2 \chi_{2} \chi_{3} \frac{1-\left(\rho_{\sigma} \rho\right)^{i-2}}{1-\rho_{\sigma} \rho}+2 \chi_{2} \chi_{4} \frac{1-\left(\rho_{\sigma} \rho^{2}\right)^{i-2}}{1-\rho_{\sigma} \rho^{2}}+2 \chi_{3} \chi_{4} \frac{1-\rho^{3(i-2)}}{1-\rho^{3}}
\end{array}\right) \\
+\chi_{2}^{2}\left((i-2)+2 \chi_{5} \frac{1-\rho_{\sigma}^{i-2}}{1-\rho_{\sigma}}+\chi_{5}^{2} \frac{1-\rho_{\sigma}^{2(i-2)}}{1-\rho_{\sigma}^{2}}\right) \\
+2 \varkappa_{1} \varkappa_{2}\left(\begin{array}{c}
(i-2) \chi_{1}+\left(\chi_{2}+\chi_{1} \chi_{5}\right) \frac{1-\rho_{\sigma}^{i-2}}{1-\rho_{\sigma}}+\chi_{3} \frac{1-\rho^{i-2}}{1-\rho}+\chi_{4} \frac{1-\rho^{2(i-2)}}{1-\rho^{2}} \\
+\chi_{2} \chi_{5} \frac{1-\rho_{\sigma}^{2(i-2)}}{1-\rho_{\sigma}^{2}}+\chi_{3} \chi_{5} \frac{1-\left(\rho_{\sigma} \rho\right)^{i-2}}{1-\rho_{\sigma} \rho}+\chi_{4} \chi_{5} \frac{1-\left(\rho_{\sigma} \rho^{2}\right)^{i-2}}{1-\rho_{\sigma} \rho^{2}}
\end{array}\right),
\end{gathered}
$$


Bringing back the final term in B.7 means that

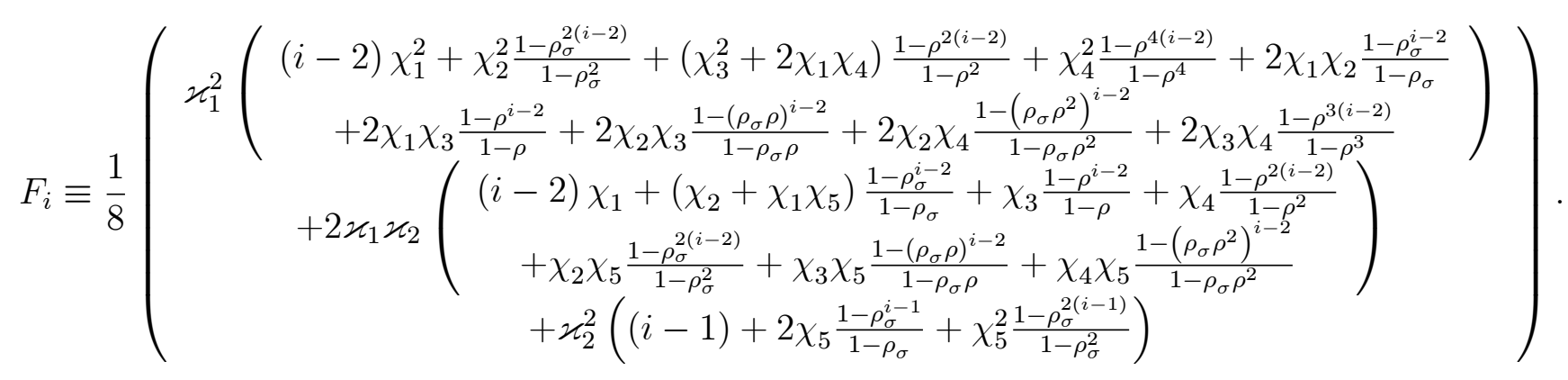

\section{B.5 Model implied data moments}

This subsection examines the model's ability to match stylized asset pricing facts. Table B.1 presents various calibrations of the model's parameters and the corresponding moments from the model solution. The moments I report are the price-dividend ratio, the risk-free rate and the equity risk premium, conditional on $\left(x_{t}, \eta_{t}\right)=(x, \eta)$. Thus, these model moments are not the analogous unconditional means in the data. ${ }^{13}$ To provide a guide to the data on asset prices, I report the unconditional mean risk-free rate and equity risk premium value that Bansal and Yaron (2004) match. The risk-free rate is a little below $1 \%$ and the equity risk premium is a little over 600 basis points.

For this exercise, I hold $\beta, x$, and $\eta$ at the values used in the main text. Rows 1-3 present the model without stochastic volatility (i.e. Burnside (1998)). A comparison of rows 1 and 2 show the standard risk-free rate and equity premium puzzles (see Mehra and Prescott (1985) and Weil (1989)). First, it is clear that the risk-aversion parameter, $\gamma$, has to be significantly greater than 11 to generate a 600 basis point equity premium. Second, as one does increase $\gamma$, the risk-free rate becomes counterfactually large.

Rows 4-6 add stochastic volatility. Using the parameter value for the stochastic volatility process from Bansal and Yaron (2004) to solve the model has no impact on the moments relative to the no-stochastic volatility case (rows 1-2 and 4-5 are virtually identical).

Rows 7-10 ask whether there are any parameter values that can generate a reasonable equity risk premium. Row 9 shows that with a risk aversion parameter of 11 , the model can generate a reasonable equity risk premium if the standard deviation of the stochastic volatility process, $\omega$ is three orders of magnitude larger than the benchmark value. This value can be reduced somewhat by making the persistence parameter, $\rho_{\eta}$ large and negative. While none of the rows 7-9 produce a risk-free rate close to the data, the model with stochastic volatility does have a risk-free rate significantly lower than, for example, rows 1 and 2. By increasing stochastic volatility, the equity risk premium increases be-

\footnotetext{
${ }^{13}$ For the price-dividend ratio and the risk-free rate - for which we do have closed-form unconditional mean expressions - the differences between those unconditional means and the ones reported in the table are not of first order importance.
} 
cause the risk-free rate falls whereas when we use the risk-aversion parameter to generate a reasonable equity risk premium, the risk-free rate is also large. Thus, the addition of stochastic volatility goes in the right direction of resolving the two asset pricing puzzles. However, with CRRA preferences, the effects are simply not powerful enough.

Finally, rows 11-12 use a gamma distribution in which the mean and variance of the stochastic volatility process are the same as rows 6 and 10 respectively. However, unlike the Gaussian distribution, the gamma distribution generates positive skew (a value of 4.77) and excess kurtosis (a value of 34.1). Relative to the Gaussian distribution, the model with the gamma distribution generates a higher price-dividend ratio and a lower risk-free rate. Thus, the gamma distribution moves the model moments towards the data moments. 
Table B.1: Model implied data moments

\begin{tabular}{|c|c|c|c|c|c|c|c|}
\hline \multicolumn{4}{|c|}{ Parameters } & & \multicolumn{3}{|c|}{ Moments } \\
\hline$\gamma$ & $\rho$ & $\rho_{\eta}$ & $\omega$ & Data: & $y$ & $\begin{array}{c}\text { rf }(\%) \\
0.86\end{array}$ & $\begin{array}{c}\text { erp (bp) } \\
633\end{array}$ \\
\hline
\end{tabular}

No Stochastic Volatility:

$\begin{array}{lccccccc}\text { 1. } & 2.5 & 0 & - & - & 12.53 & 9.67 & 33 \\ \text { 2. } & 11 & 0 & - & - & 5.39 & 19.19 & 158 \\ 3 . & 2.5 & 0.7 & - & - & 14.63 & 9.67 & -61\end{array}$

Stochastic Volatility:

$\begin{array}{cccccccc}\text { 4. } & 2.5 & 0 & 0 & \times 1 & 12.53 & 9.67 & 33 \\ 5 . & 11 & 0 & 0.855 & \times 1 & 5.39 & 19.20 & 158 \\ 6 . & 11 & 0 & 0 & \times 500 & 5.94 & 16.25 & 278\end{array}$

\section{Targeting the ERP:}

$\begin{array}{cccccccc}7 . & 2.5 & 0 & 0 & \times 15000 & 13.89 & 3.27 & 610 \\ 8 . & 2.5 & -0.2 & 0 & \times 14500 & 13.12 & 3.67 & 636 \\ 9 . & 11 & 0 & 0 & \times 1100 & 10.07 & 5.58 & 640 \\ 10 . & 11 & 0 & -0.9 & \times 650 & 5.77 & 14.25 & 625\end{array}$

\section{Gamma distribution:}

$\begin{array}{lccccccc}\text { 11. } & 11 & 0 & 0 & \times 500 & 6.58 & 13.31 & - \\ \text { 12. } & 11 & 0 & -0.9 & \times 650 & 6.24 & 3.49 & -\end{array}$

Note: The parameters $\beta, x, \eta$ are held constant at $.95, .0179$, and .0012, respectively. rf and erp denote the risk-free rate and equity risk premium, respectively, and bp denotes basis points. The moments are evaluated at $\left(x_{t}, \eta_{t}\right)=(x, \eta)$. Data estimates from Bansal and Yaron (2004). The column for $\omega$ reports multiples of the benchmark calibrated value, $\omega=0.75 \times 10^{-5}$. The gamma distribution has the following parameters: $\omega^{\Gamma}=\omega, \eta^{\Gamma}=0, g_{1}=2.3855, g_{2}=0.1757$. 